\title{
Role of Endogenous Vintage Specific Depreciation in the Optimal Behavior of Firms
}

\author{
Cagri Saglam* and Vladimir M. Veliov ${ }^{\dagger}$
}

\begin{abstract}
This paper studies the firms' capital accumulation process in a vintage capital model with embodied technological change. We take into account that depreciation is endogenous and in particular associated with vintage specific maintenance expenditure. We prove that maintenance is a local substitute for investment as soon as the marginal cost of maintenance is strictly increasing. We show that maintenance and investment in new capital goods appear as complements with respect to the changes in productivity, cost of maintenance, fixed cost of operation, efficiency of maintenance services and appear as substitutes with respect to the price of new machines. Allowing for investment in old vintages, we determine that investment in old machines appears as a substitute of both investments in new machines and maintenance services. We end up by analyzing the effects of technological progress on optimal plans and prove that a negative anticipation effect can occur even without any market imperfections.
\end{abstract}

Key words vintage capital, embodied technological progress, investment analysis, maintenance services, substitution versus complementarity

JEL classification D92, O33, E22, C61

Accepted 10 October 2007

\section{Introduction}

In standard models of capital accumulation, the firm owns a stock of capital goods that are equally productive and produce goods of the same quality to be sold at the market to obtain revenue. Any technological progress equally affects all capital goods, including those installed in the past. Capital goods stock increases through the investment decisions of the

\footnotetext{
*Department of Economics, Bilkent University, Ankara, Turkey. Email:csaglam@bilkent.edu.tr

${ }^{\dagger}$ Institute of Mathematical Methods in Economics, Vienna University of Technology, Vienna, Austria, and Institute of Mathematics and Informatics, Bulgarian Academy of Sciences, Sofia, Bulgaria.

The first author was financed for this research by the European Commission under the RTN Grant HPRN-CT000234-2002. Any opinions, findings and conclusions or recommendations expressed in this material are those of the authors and do not necessarily reflect the views of the European Commission. The second author was partly supported by the Austrian Science Foundation (FWF) under grant P18161, and by the Austrian National Bank (ONB) under grant Jubiläumsfond 11260.
} 
firm and decreases through depreciation at an exogenously fixed rate. However, it is now widely admitted that the assumptions according to which capital is homogeneous, technical progress is disembodied and the capital depreciation rate is exogenous and constant are to a large extent unrealistic and misleading.

Typically, technical change is increasingly embodied in the introduction of new capital goods (see Gordon 1990; Greenwood, Hercowitz, and Krussel 1997; Dekle 2001) implying that capital goods of later dates are either more productive or able to produce goods of better quality. The capital depreciation rate is endogenous, and, in particular, associated with maintenance services depending on the age of the capital goods (see, among others, Grilliches 1960; Nelson and Caputo 1997; Hamilton 1999; Whelan 2002). With this in mind, the age structure of capital turns out to be relevant for the optimal investment plans, and it is necessary to distinguish capital goods of different generations. To this end, this paper presents a vintage capital model in which firms have to decide not only on the volume of the capital goods but also on the age distribution of them under embodied technical change and endogenous vintage specific depreciation.

In a vintage capital framework, equipment of different vintages differs in its productivity due to embodied technical change (e.g. Solow, Tobin, Von Weizsacker, and Yaari 1966; Malcomson 1975; Boucekkine, Germain, and Licandro 1997; Boucekkine, Germain, Licandro, and Magnus 1998) or due to different depreciation schedules (e.g. Benhabib and Rustichini 1991). The implications of embodied technical change on the process of capital accumulation have been widely studied in the macroeconomics literature (see Boucekkine, De la Croix, and Licandro (2008) for an excellent review of the seminal contributions in this field). In particular, the productivity differential between successive vintages of capital due to embodied technical progress, often referred to as the vintage effect, has been proven to be crucial in providing answers to the questions posed by Chari and Hopenhayn (1991) regarding the investment dynamics: Why are new technologies often adopted so slowly? Why do people invest in old technologies even when apparantly superior technologies are available? How are decisions to adopt new technologies affected by the prospect that even better technologies will arrive in the future?

According to the vintage effect, younger machines are endowed with a superior technology and benefit from a longer lifetime whereas older machines are cheaper and benefit from lower costs of depreciation and discounting. This implies that a technological accelaration induces on one hand an incentive to invest in new vintages to profit from their increasing efficiency, but on the other hand, tends to reduce the profitability of investment and requires a longer upkeep of the existing equipment to equalize the marginal profit and marginal cost of investment (see Boucekkine, Germain, Licandro, and Magnus 1998). With the presence of these latter effects, although the early models of vintage differentiation (e.g. Johansen 1959; Solow 1960; Benhabib and Rustichini 1991; and the works of Boucekkine, Germain, and Licandro 1997, 1998) might provide an explanation for why new technologies are often adopted on a large scale with a substantial time delay, these contributions cannot be used to answer the second question of Chari and Hoppenhayn. A vintage capital model that allows investment in non-frontier vintages is needed. This is typically carried out by applying a partial differential equation approach for the analysis of the vintage effect (see Feichtinger, Hartl, Kort, and Veliov 2006a,b). 
Concerning the third question of Chari and Hoppenhayn, Feichtinger, Hartl, Kort, and Veliov (2006a) have figured out that the predictions of an accelaration in future technological progress do not influence the current investment decisions of a perfectly competitive firm (see Feichtinger, Hartl, Kort, and Veliov 2006a). However, by introducing market imperfections, a negative anticipation effect has been shown to prevail in the sense that the anticipated technological breakthrough precedes the declines of investment as it will be optimal for the firm to delay investment in new machines till the breakthrough (e.g. Feichtinger, Hartl, Kort, and Veliov 2006b, 2007). In the case of a market power, because current investments increase output, which in turn decreases price, this creates a negative effect on the profitability of future investments, so that the anticipated technology shocks will be accompanied by initial declines of investment. This is followed by a period of higher growth where new capital goods can be purchased without reducing the output price too much.

Despite the relatively large published literature on vintage models concerned with the effects of technical change, scant attention has been paid to the endogenous nature of the depreciation rate depending on the vintage specific maintenance activities. These studies that assume a stable depreciation pattern are likely to miss a major component of the capital accumulation process and, as recently demonstrated by Laitner and Stolyarov (2003) and Mukoyama (2007), can even lead to potentially flawed measurement of capital stock, especially in periods of rapid technical progress.

An acceleration in embodied technical change implies the continuous introduction of new capital goods that are more productive than those already in place. The cost of capital services from the new vintages would be continuously lowered, exhausting the quasi-rents of the older capital goods so that maintenance of the older capital goods would eventually cease to be economical. In this sense, as carefully evidenced by Bitros and Kelejian (1974) and Bitros (1976), the decision to alter the useful life of existing capital stock through maintenance services affects the decision to invest in new capital goods. Similarly, McGrattan and Schmitz (1999) and, more recently Mullen and Williams (2004), examining data from an economywide survey in Canada, show that maintenance activity is large relative to investment expenditures and to some extent a substitute for investment. In accordance with these studies, the analysis of the trade-offs that exist among maintenance services, and investment in new and old vintages of capital goods raises the following questions and are crucial for a better understanding of the firm's capital accumulation process:

(i) Under what conditions do maintenance and investment appear as substitutes or complements?

(ii) What is the optimal allocation of investment and maintenance across vintages?

(iii) How do maintenance and investment decisions shift in response to an exogenous change in the rate of technological progress?

(iv) Can there be a negative anticipation effect even under non-monopolistic settings?

Among the very few early attempts to provide answers to these questions, Hartl (1983) and Boucekkine and Ruiz-Tamarit (2003) investigate the demand for maintenance services in some typical firms' investment problems and analyze the substitutability of these services 
with investment. However, these papers, assuming a homogenous stock of capital goods, could not explore the linkages between investment and maintenance expenditures directed towards each available vintage and, hence, provide answers to the questions (ii)-(iv). Boucekkine, Rio, and Martinez (2005), concerned with the economic performances at the aggregate level, analyze question (iii) and determine that an increase in embodied technical progress increases the depreciation rate, which in turn reduces the operating lifetime of capital goods. As the analysis does not allow for the possibility to invest in older capital goods, the trade-off between investing in new versus the existing capital goods and the maintenance of these goods have not been taken into consideration. Along this line, Boucekkine, Martinez, and Saglam (2006) analyze the optimal allocation of labor resources to production, technology adoption or capital maintenance in a one-hoss-shay vintage capital model and point out that although capital maintenance deepens the technological gap by diverting labor resources from adoption, it generally increases the long-run output level at equilibrium.

Apart from these studies, the theoretical contributions to the role of maintenance in economics are mostly composed of empirical or computational analyses of the cyclical properties of maintenance and its implications for the business cycle (see, among others, Licandro and Puch 2000; Collard and Kollintzas 2000). As a result, the questions regarding the analysis of the optimal maintenance expenditures and the interactions of such expenditures with technological progress and the investment profiles in a vintage capital model remain unabated.

In this paper, we provide the needed rigorous analysis of the firm's capital accumulation process taking into account that depreciation is endogenous and, in particular, associated with vintage specific maintenance expenditure. We present precise answers to questions (i) -(iv) with a detailed analysis of the substitutability between investment and maintenance services. We provide an intrinsic definition of complementarity versus substitutability and prove that maintenance is a local substitute for investment as soon as the marginal cost of maintenance is strictly increasing. We show that maintenance and investment in new capital goods appear as complements with respect to the changes in productivity, cost of maintenance, fixed cost of operation and the efficiency of maintenance services. We also find that investment in new capital goods and maintenance services are substitutes in the traditional sense: when the price of new machines changes, the demands for capital goods and for maintenance respond in the opposite direction. We analyze the effects of technological progress on the firm's optimal plans and prove that a negative anticipation effect can occur even without any market imperfections. Our set up allows for an extension where investments in the old vintages are possible. We show that investment in old machines appears as a substitute for both investments in new machines and maintenance services.

Methodologically this paper builds on the capital accumulation models investigated in Barucci and Gozzi (1998) and Feichtinger, Hartl, Kort, and Veliov (2006a,b) by introducing maintenance as a control variable. The model fits the general framework in Feichtinger, Tragler, and Veliov (2003) and the analysis uses the optimality conditions obtained there. A methodological novelty in the context of vintage models with partial differential equations (PDE) is the possibility to interpret the controlled depreciation rate as a scrapping time control, as explained in Remark 2 in Section 2. Technically, the paper is substantially more 
complicated than Feichtinger, Hartl, Kort, and Veliov (2006a,b) due to the nonlinearity in the dynamics brought by the maintenance control. A remarkable feature of our analysis is that it does not rely on particular parameterizations of the exogenous functions involved in the model; rather, it uses only general and plausible qualitative properties. Therefore, the obtained results are robust.

The paper is organized as follows. Section 2 describes the model. Section 3 presents the optimality conditions and the main assumptions of the analysis. In Section 4 we conduct comparative economic analysis and aim to answer whether investments and maintenance appear as complements or substitutes with respect to the variations in different exogenous factors. We also make the analysis under an intrinsic definition of substitutability. Section 5 analyzes the negative anticipation effect of the technological progress and presents numerical illustrations. Section 6 extends the model to allow for investment in old machines and discusses the implications. Some more technical proofs are provided in the Appendix.

\section{The Model}

In this section we present our model, which has the form of an age-structured PDE optimal control system with a main dynamic equation similar to that introduced in Barucci and Gozzi (1998). The time is continuous, and for convenience we consider a finite (but 'large') horizon $[0, T]$. For any time $t$, we denote by $K(t, a)$ the number of machines of age $a$ that are in use by the firm. ${ }^{1}$ Each machine has a fixed maximal lifetime $\omega$. The productivity of the machines build at time $t$ is $f(t)$, where it is assumed that due to embodied technological progress, the new generations of machines are more productive than the old ones; that is, $f$ is increasing. Following Feichtinger, Hartl, Kort, and Veliov (2006a), the total output produced at time $t$ by the firm is defined as

$$
Q(t)=\int_{0}^{\omega} f(t-a) K(t, a) \mathrm{d} a,
$$

where $f(t-a)$ is the productivity of the machines that at time $t$ are of age $a$; that is, the productivity of the machines of technology vintage $t-a$. The firm's revenue, is then $p Q(t)$, where $p$ is the output price. ${ }^{2}$

When describing the evolution of the capital stock we take into account the dependence of the capital depreciation on age and on maintenence. The depreciation is typically larger for old capital goods (see e.g. Boucekkine, De la Croix, and Licandro 2004; Feichtinger, Hartl, Kort, and Veliov, 2006b). Moreover, it is far from convincing that the capital depreciation rate is exogenous, especially at the firm level (see McGrattan and Schmitz 1999; Boucekkine and Ruiz-Tamarit 2003). Firms typically control the depreciation rate of capital by choosing

\footnotetext{
${ }^{1}$ Here "number" is a colloquial term. Strictly speaking, $K(t, \cdot)$ is a (non-probabilistic) density, so that $\int_{a_{1}}^{a_{2}} K(t, a) \mathrm{d} a$ is the number of machines of age between $a_{1}$ and $a_{2}$.

${ }^{2}$ An output-dependent price $p=p(Q(t))$ could also be investigated in the same framework, following Feichtinger, Hartl, Kort, and Veliov (2006b). However, in this paper we assume perfect competition in order to focus on the effects caused by the maintenance, rather than of the output dependence of price, which is investigated in the abovementioned paper.
} 
an appropriate level of vintage-specific maintenance services. Therefore, we consider the level of maintenance services that the firm chooses for the machines of age $a$ at time $t$, denoted by $m(t, a)$, as a control variable. It is clear that the more resources devoted to maintenance, the lower the depreciation rate. Hence, the evolution law of capital stock is described by

$$
K_{t}+K_{a}=-\delta(a, m(t, a)) K(t, a), \quad K(0, a)=K_{0}(a), \quad K(t, 0)=I(t),
$$

where the subscripts denote the partial differentiation, $I(t)$ is the inflow of new machines that the firm purchases at time $t, K_{0}(a)$ is the given initial data for the firm's capital, and $\delta$ is the physical depreciation rate, depending on the maintenance level. This specification is based on the fact that time and age move together: machines aged $a+\mathrm{d} t$ at time $t+\mathrm{d} t$ are those aged $a$ at time $t$, minus their depreciation, plus capital goods of this vintage invested at time $t$. In the present basic version of the model we do not include the possibility of investing in non-frontier vintages, for the sake of simplicity. Section 6 discusses some consequences of this possibility. The strict meaning of the solution of the above equation is given in, for example, Feichtinger et al.(2003) and Webb (1985).

We denote by $C(I)$ the total (acquisition and implementation) cost of installing $I$ new machines. Although $C(I)$ is typically a linear-quadratic function, in our analysis it may have a rather general form. Notice that $C(I)$ is assumed independent of time, which means that although the newer machines become more productive due to the technological progress, the acquisition cost remains the same. A typical example is the computer industry (see Hulten and Wykoff 1995).

The cost of maintenance services is represented by $D(M)$, where $M$ accounts for the total maintenance services at time $t$,

$$
M(t)=\int_{0}^{\omega}\left(m_{0}+m(t, a)\right) K(t, a) \mathrm{d} a,
$$

given the fact that as long as a machine is in operation it requires a fixed maintenance (or operation) cost, $m_{0} \geq 0$, in addition to the controlled maintenance level, $m(t, a)$, which is a matter of the firm's choice.

The firm maximizes the discounted value of the cash flow over the planning horizon $[0, T]$. Denoting the discount factor by $r$, we obtain the following age-specific dynamic optimization model of the firm:

$$
\max _{I, m} \int_{0}^{T} e^{-r t}[p Q(t)-C(I(t))-D(M(t))] \mathrm{d} t
$$

subject to

$$
\begin{gathered}
K_{t}+K_{a}=-\delta(a, m(t, a)) K(t, a), \quad K(0, a)=K_{0}(a), \quad K(t, 0)=I(t), \\
Q(t)=\int_{0}^{\omega} f(t-a) K(t, a) \mathrm{d} a
\end{gathered}
$$




$$
\begin{gathered}
M(t)=\int_{0}^{\omega}\left(m_{0}+m(t, a)\right) K(t, a) \mathrm{d} a, \\
m(t, a) \geq 0 \\
I(t) \geq 0 .
\end{gathered}
$$

Remark 1 We consider a finite planning horizon in the above model to avoid complications with the optimality conditions, as explained in Remark 3 in the next section. This is not a drawback, because we do not perform an asymptotic analysis, focusing on present/intermediate effects. Indeed, a typical property of the class of vintage models with bounded scrapping age and marginally increasing investment costs is that the influence of the length of the planning horizon, $T$, on the behavior of the optimal solution on a fixed finite time-interval becomes negligible when $T$ is sufficiently large, which we presume. To our knowledge, this important property, although supported by numerical (including our case studies) and by some formal arguments, has not been proven so far, and presents a mathematical challenge that will be addressed by the second author in a collaboration paper in preparation.

\section{Standing assumptions:}

(i) $C, D:[0, \infty] \mapsto[0, \infty]$ have continuous second derivatives, and $C(0)=0, C^{\prime}(I)>0$, $C^{\prime \prime}(I) \geq \gamma^{C}>0, D(0)=0, D^{\prime}(M) \geq \gamma^{D}>0, D^{\prime \prime}(M) \geq 0$

(ii) $\delta:[0, \omega] \times[0, \infty] \mapsto[0, \infty]$ is twice differentiable in $m$ with Lipschitz continuous $\delta_{m}^{\prime}$ and $\delta_{m m}^{\prime \prime}$, and $\delta_{m}^{\prime}(a, m)<0, \delta_{m m}^{\prime \prime}>0$;

(iii) $f:[-\omega, T] \mapsto(0, \infty)$ is piecewise continuous and non-decreasing;

(iv) $K_{0}:[0, \omega] \mapsto[0, \infty)$ is continuous, $m_{0} \geq 0, r \geq 0, p>0$.

We stress that the principle assumptions, namely that $C$ and $\delta$ are strongly convex (the second one with respect to $m$ ), are plausible. The first one is standard, while the second means that the marginal efficiency of maintenance decreases. For the function

$$
\delta(a, m)=\bar{\delta}(a)+\frac{1}{\alpha+\beta m}, \quad \alpha, \beta>0,
$$

considered in Boucekkine and Ruiz-Tamarit (2003), for example, assumption (ii) is apparently fulfilled. Here $\bar{\delta}(a)$ (presumably increasing) is the lower bound for the depreciation rate at age $a$, while $\bar{\delta}(a)+1 / \alpha$ is the depreciation rate without maintenance.

Remark 2 The involvement of a maintenance control can be viewed as a way of endogenizing the scrapping time. Indeed, using a depreciation function such as $\delta(a, m)=\bar{\delta}(a)+\tilde{\delta}(a, m)$ with $\tilde{\delta}(a, 0)=0$ and $\tilde{\delta}(a, \infty)=\infty$ one may view $m$ as a scrapping control (the higher is the value of $m$ the more machines are scrapped), while $M(t)$ is interpreted as the cost of scrapping, if any. An alternative way (where $m$ is still considered as maintenance), is to take a small parameter $\alpha>0$ in (7), in which case the machines of the respective age would be quickly scrapped in the absence of maintenance. 
Proposition 1 The problem (1)-(6) has a solution.

The proof (which is not obvious) is sketched in the Appendix. We stress that the solution for $m$ need not be unique. Indeed, if for some $t$ and $a$ it happens that the optimal $K(t, a)=0$ then the value of $m(t, a)$ does not matter. At such points (if any) we set $m(t, a)=0$.

\section{Optimality conditions}

To solve the problem (1)-(6) we apply the maximum principle for general age-structured optimal control problems obtained in Feichtinger et al. (2003). We mention that the earlier optimality conditions (see Brokate 1985) do not fit the problem due to the presence of the control $m$ in both the differential equation (2) and the integral expression (4). The maximum principle in Feichtinger et al. (2003) implies the following.

Proposition 2 Let $I, m, K, Q, M$ be an optimal solution of problem (1)-(6). Then the adjoint equation

$$
\begin{gathered}
\xi_{t}+\xi_{a}=(r+\delta(a, m(t, a))) \xi-p f(t-a)+D^{\prime}(M(t))\left(m_{0}+m(t, a)\right), \\
\xi(t, \omega)=0, \quad \xi(T, a)=0
\end{gathered}
$$

has a unique solution $\xi$, and

$$
\begin{gathered}
\xi(t, 0) I(t)-C(I(t))=\max _{I \geq 0}\{\xi(t, 0) I-C(I)\} \quad \text { for almostall }(t, a), \\
\xi(t, a) \delta(a, m(t, a))+D^{\prime}(M(t)) m(t, a)=\min _{m \geq 0}\left\{\xi(t, a) \delta(a, m)+D^{\prime}(M(t)) m\right\} \\
\text { for almost all }(t, a) \text { for which } K(t, a) \neq 0 .
\end{gathered}
$$

Remark 3 The zero end condition for the adjoint variable is a "privilege" of the finite planning horizon. In the case $T=\infty$ a transversality condition at infinity would be needed to obtain a complete system of necessary conditions. To our knowledge such a condition is not known for age-structured controlled PDE with non-local terms entering in the dynamics or (in a nonlinear way) in the objective function. The authors are in favor of the following transversality condition "at infinity": $\xi$ is bounded. For ordinary equations a condition of this type is known from Aubin and Clarke (1979) for linear systems, extended recently in Aseev and Kryazhimskiy (2004). For PDE vintage models the above transversality condition is formulated in several papers by the second author, but a proof that it uniquely determines the solution of the adjoint system is given only in Feichtinger and Veliov (2007), in a situation that does not cover the present one. Therefore, in the present paper we prefer the finite horizon formulation.

Because the optimal solution in the interval $[0, \omega]$ depends on the initial data $K_{0}(a)$ (which are in a sense arbitrary), and in the interval $[T-\omega, T]$ it may be distorted by 
the forthcoming end of the planing horizon (see Remark 2), the economically meaningful analysis in the next section will be restricted to the time interval $[\omega, T-\omega]$.

The following assumption and lemma provide a more explicit representation of the optimal controls from the maximum principle.

Regularity assumption:

The following inequality is fulfilled for the optimal maintenance costs:

$$
p f(t-\omega)>D^{\prime}(M(t)) m_{0} \text { for all } t \in[0, T] .
$$

Inequality (11) means that the marginal revenue obtained from the oldest machines in use exceeds the marginal expenditure of maintaining the machines at the minimal required level.

Lemma 1 For the solution $\xi$ of the adjoint equation (8) it holds that $\xi(t, a)>0$ for every $t$ $\in(\omega, T)$ and $a \in[0, \omega)$.

Thanks to the above lemma the optimality conditions (9) and (10) can be rewritten in the following more convenient form. Define the functions

$$
\begin{gathered}
\left(C^{\prime}\right)_{+}^{-1}(y)= \begin{cases}\left(C^{\prime}\right)^{-1}(y) & \text { if } y \geq C^{\prime}(0), \\
0 & \text { else, }\end{cases} \\
\left(\delta_{m}^{\prime}\right)_{+}^{-1}(a, y)= \begin{cases}\left(\delta_{m}^{\prime}\right)^{-1}(a, y) & \text { if } y \geq \delta_{m}^{\prime}(a, 0), \\
0 & \text { else, }\end{cases}
\end{gathered}
$$

where $\left(C^{\prime}\right)^{-1}$ and $\left(\delta_{m}^{\prime}\right)^{-1}$ denote the inverse functions of the corresponding derivatives (with respect to $m$ for $\delta_{m}^{\prime}$ ). Then the optimal investment and maintenance can be expressed from (9) and (10) as

$$
I(t)=\left(C^{\prime}\right)_{+}^{-1}(\xi(t, 0)), \quad m(t, a)=\left(\delta_{m}^{\prime}\right)_{+}^{-1}\left(a,-\frac{D^{\prime}(M(t))}{\xi(t, a)}\right) .
$$

Remark 4 It is useful to notice that in $(12)\left(C^{\prime}\right)_{+}^{-1}$ is increasing with respect to $\xi(t, 0)$ and $\left(\delta_{m}^{\prime}\right)_{+}^{-1}$ is increasing with respect to $\xi(t, a)$. The assumptions for $C, D$ and $\delta$ imply that at points where $I$ (respectively, $m$ ) is positive, the increase is strict.

Let us denote

$$
\hat{m}(a, M, \xi)=\left(\delta_{m}^{\prime}\right)_{+}^{-1}\left(a,-\frac{D^{\prime}(M)}{\xi}\right) .
$$

With this feedback low the adjoint equation becomes

$$
\xi_{t}+\xi_{a}=(r+\delta(a, \hat{m}(a, M(t), \xi))) \xi-p f(t-a)+D^{\prime}(M(t))\left(m_{0}+\hat{m}(a, M(t), \xi)\right) .
$$

The right-hand side is well-defined and Lipschitz continuous in $\xi \geq 0$. Indeed, if $\xi$ is close to zero, then $-\frac{D^{\prime}(M)}{\xi}<\delta_{m}^{\prime}(a, 0)$ due to the assumption that $D^{\prime}(M) \geq \gamma^{D}>0$; hence, 
$\hat{m}(a, M, \xi)=0$. The other points of non-differentiability of $\hat{m}$ in $\xi$ are caused by the operation of maximuminvolved in $\left(\delta_{m}^{\prime}\right)_{+}^{-1}$, which does not spoil the Lipschitz continuity. In particular, (14) has a unique solution, which necessarily coincides with the one of (8).

\section{Comparative economic analysis}

In this section we investigate how the optimal investment and maintenance depend on different exogenous factors involved in the model. In some of the considerations below we assume that the maintenance cost, $D(M)$, depends linearly on the total maintenance and operation services; that is, $D(M)=d M$ ( $d>0$ is a constant). This is a substantial simplification, because in this case the adjoint equation (14) completely decouples from the state equation and can be investigated separately. Namely, it takes the form

$$
\begin{aligned}
& \xi_{t}+\xi_{a}=(r+\delta(a, \hat{m}(a, \xi))) \xi-p f(t-a)+d\left(m_{0}+\hat{m}(a, \xi)\right) \\
& \xi(t, \omega)=0, \quad \xi(T, a)=0
\end{aligned}
$$

where now we skip the argument $M(t)$ in the notation $\hat{m}(a, M, \xi)$, since the latter is independent of $M$. As Remark 6 shows, the assumption for linearity of the maintenance cost is essential for the validity of the next proposition. However, the two most interesting statements below are proved in the case of a general nonlinear maintenance cost function. In what follows it is supposed that the standing and the regularity assumptions hold for the sets of data involved in the considerations. Also, the linearity condition $D(M)=d M$ is assumed in this section, unless an alternative condition is specifically formulated.

Proposition 3 Consider two technology functions $f_{1}$ and $f_{2}$ for which $f_{1}(t)<f_{2}(t)$ at a time $t \in[0, T-\omega]$. Then for the corresponding optimal solutions, $\left(I_{1}, m_{1}\right)$ and $\left(I_{2}, m_{2}\right)$, it holds that

$$
I_{1}(t) \leq I_{2}(t), \quad m_{1}(t+a, a) \leq m_{2}(t+a, a) \text { for every } a \in[0, \omega] .
$$

Moreover, each of the inequalities is strict, unless $I_{1}(t)=I_{2}(t)=0$ or $m_{1}(t, a)=m_{2}(t, a)=0$, respectively.

According to this proposition, investment and maintenance appear as complements with respect to productivity. It is optimal for a firm to invest more in technologies of higher productivity and also to better maintain these vintages.

The proof of the above proposition is simple; therefore, we present it in the main text.

Proof of Proposition 3: On every characteristic line starting at the point $(t, 0)$ (i.e. on the segment $\{(t+s, s): s \in[0, \omega]\})$ the solution $\xi$ of the adjoint equation (15) satisfies an ordinary differential equation. Namely, $\eta(s)=\xi(t+s, s)$ satisfies

$$
\eta^{\prime}(s)=\left(r+\delta(s, \hat{m}(s, \eta(s))) \eta(s)-p f(t)+d\left(m_{0}+\hat{m}(s, \eta(s))\right),\right.
$$


with $\eta(\omega)=0$. We have to compare the solutions $\eta_{1}$ and $\eta_{2}$ of this equation with $f_{1}$ and $f_{2}$ substituted for $f$. If $\eta_{1}=\eta_{2}$, then the difference of the right-hand sides is

$$
\eta_{2}^{\prime}-\eta_{1}^{\prime}=-p\left(f_{2}(t)-f_{1}(t)\right)<0 .
$$

Because $\eta_{1}(\omega)=\eta_{2}(\omega)=0$, this implies that $\eta_{2}(s)>\eta_{1}(s)$ for $s \in[0, \omega)$. Then the formulas in (12) and Remark 4 imply the claims of the proposition.

Corollary 1 Let $\bar{a}_{i}(t)(i=1,2)$ be the maximal age of maintaining machines at time $t$ for the technology function $f_{i}$; that is, $m_{i}(t, a)=0$ for $a \geq \bar{a}_{i}(t)$, but $m_{i}(t, a-\varepsilon)>0$. Assume that $\bar{a}_{2}(t)>0$. Then

$$
\bar{a}_{1}(t)<\bar{a}_{2}(t) .
$$

The maximal age of the machines to be maintained would be higher for the more productive technologies. Even if it is technologically feasible to maintain a machine till the end of its maximal life-time, it ceases to make economic sense at some point because the upkeep ultimately becomes more expensive compared with the cost of a new and superior machine (see Gylfason and Zoega 2001). This sheds some light on the rapid disappearance of early models in the computer industry. As pointed out by Geske, Ramey, and Shapiro (2004), at an age of 1 year, the RAM of a used computer is 48 percent below the median RAM of a new computer, its speed is 36 percent slower and its hard disk is 52 percent smaller. For older ages, the decline is rapid and continues to fall. The new computer models are typically more productive and are, therefore, maintained more relative to the older ones.

Proposition 4 Consider two prices of maintenance, $d_{1}<d_{2}$. Then for the corresponding optimal solutions, $\left(I_{1}, m_{1}\right)$ and $\left(I_{2}, m_{2}\right)$, it holds that

$$
I_{1}(t) \geq I_{2}(t), \quad m_{1}(t, a) \geq m_{2}(t, a) \text { for every } a \in[0, \omega] .
$$

Moreover, each of the inequalities is strict, unless $I_{1}(t)=I_{2}(t)=0$ or $m_{1}(t, a)=m_{2}(t$, a) $=0$, respectively.

The proof of this proposition uses exactly the same argument as the previous one (excepting the case $m_{0}=0$, which requires some more routine work); therefore, we skip it.

Corollary 1 applies also to Proposition 4. Exactly the same claim as in Proposition 4 holds for the dependence of the optimal solution on the fixed cost of operation, $m_{0}$.

Investment and maintenance appear as complements with respect to the price of maintenance and with respect to the fixed costs of operation. Remembering the empirical assessment of the economy-wide importance of the maintenance costs in McGrattan and Schmitz (1999) (approximately 6 percent of the Canadian gross domestic product), this proposition becomes vital in exploring the firm's optimal plans analytically. An increase in the price of maintenance services leads firms to lower their demand for both investment goods and maintenance services. Thus, in accordance with Boucekkine and Ruiz-Tamarit (2003), instead of finding that maintenance is a substitute for investment expenditures, 
we found that investment and maintenance behave as gross complements. This confirms the empirical findings of Mullen and Williams (2004) that the investment has a positive and statistically significant impact on the maintenance spending. The interpretation is that investment acts as a proxy for demand conditions that exert a direct impact on the desire to maintain existing stock. An increase in the price of maintenance leads to a decrease in both the maintenance and the investment activities because of an income effect, and leads to a decrease in the maximal age of the machines to be maintained because of a substitution effect. These results reinforce the empirical findings of Parks (1977) that suggest that the increase in the relative cost of maintenance was the source of rising scrapping rates between 1947 and 1969 for domestically produced post-war vintage automobiles in the USA.

According to Proposition 4, investment and maintenance appear as complements also with respect to the fixed costs of operation. Accordingly, the maximal age of the machines to be maintained decreases with the increasing fixed costs of operation and with the price of maintenance. It is important to note that the maintenance is lower when the cost of operation is higher. The fixed cost assumption is motivated by empirical observations. For instance, the increase in demand for IT positions is a clear indication of the need to backup computer investments with outlays on maintenance and support (see Whealan 2002). The fixed cost of operation induces the phenomenon of obsolescence. Once the marginal productivity of a machine falls behind the fixed cost of operation the firm will choose not to maintain the machine anymore. These results explain why older models disappear more rapidly from the computer industry market, especially in comparison with the auto industry. One car for $\$ 50000$ is operated by one person, one PC for $\$ 1000$ by one person. If the salaries of the two workers are approximately the same (which are included in the cost of operation), then $m_{0}$ for computers is 50 times larger than that for cars (per input price), leading to a lower level of maintenance and, therefore, higher depreciation rates for computers.

Proposition 5 Consider two depreciation functions $\delta_{1}$ and $\delta_{2}$ for which the standing assumptions hold, and additionally that $\delta_{1}$ and $\delta_{2}$ are related in the following way: for every a $\in[0, \omega]$ and $m \geq 0$ (i) $\delta_{1}(a, m) \leq \delta_{2}(a, m)$; (ii) $\delta_{1, m}^{\prime}(a, 0) \leq \delta_{2, m}^{\prime}(a, 0)$, where $\delta_{i, m}^{\prime}$ is the derivative of $\delta_{i}$ with respect to $m$. Then for the corresponding optimal solutions, $\left(I_{1}, m_{1}\right)$ and $\left(I_{2}, m_{2}\right)$, it holds that

$$
I_{1}(t) \geq I_{2}(t), \quad m_{1}(t, a) \geq m_{2}(t, a) \text { for every } a \in[0, \omega]
$$

Moreover, if the inequality in (i) is strict for $a>0$, then each of the inequalities (17) is also strict, unless $I_{1}(t)=I_{2}(t)=0$ or $m_{1}(t, a)=m_{2}(t, a)=0$, respectively.

The proof uses once again a comparison argument for (16), but requires some more technical arguments, which are presented in the Appendix.

Investment and maintenance appear to be complements also with respect to the efficiency of maintenance. This means that a higher depreciation rate for the same maintenance level leads to less investment and maintenance. However, the situation is different for the 
dependence of the optimal investment and maintenance on the acquisition cost of machines.

Proposition 6 Consider two costs functions for new capital, $C_{1}$ and $C_{2}$, where $C_{1}^{\prime}(I)<C_{2}^{\prime}(I)$ for all $I>0$. Then for the corresponding optimal solutions, $\left(I_{1}, m_{1}\right)$ and $\left(I_{2}, m_{2}\right)$, it holds that

$$
I_{1}(t) \geq I_{2}(t), \quad m_{1}(t, a)=m_{2}(t, a) \text { for every } a \in[0, \omega] .
$$

Moreover, the inequality is strict, unless $I_{1}(t)=I_{2}(t)=0$.

For a proof it is enough to notice that the adjoint Equation (15) does not depend on the function $C$; therefore, the formulae for $I$ and $m$ in (12) imply the claim.

Remark 5 The independence of the maintenance on the price of the new machines is heavily bound with the assumption of linearity of the maintenance cost. If for the cost-ofmaintenance function, $D(M)$, it holds that $D^{\prime \prime}(M) \geq \rho>0$ for every $M \geq 0$, then

$$
m_{1}(t, a)<m_{2}(t, a) \text { for some }(t, a) \in[\omega, T-\omega] \times[0, \omega]
$$

provided that $m_{2}(t, a)$ is not identically zero. The proof of this claim is much more involved and we do not present it.

The decision on whether to maintain an existing machine or let it go to scrap has to be made using the marginal costs of new machines. This issue has been the subject of many empirical studies (see, among others, Schmalensee 1974; Parks 1979; Berkovec 1985; Nelson and Caputo 1997). In a recent paper, Hamilton and Macauley (1999) disentangle the source of the dramatic increase in the longevity of automobiles over the past 25 years and conclude that, with the increase in new car prices and the decrease in the expense of auto maintenance, it is optimal to increase the maintenance to an older age. However, among the very few analytical contributions, Boucekkine and Ruiz-Tamarit (2003), with a homogeneous stock of capital goods assumption, determine that maintenance and investment are complementary, even with respect to the acquisition cost of machines. In this respect, Proposition 6 and Remark 5 analytically confirm the empirical findings and prove crucial in identifying the effect of the acquisition costs of machines on the scrapping rates and the link between investment and maintenance. In contrast to Boucekkine and Ruiz-Tamarit (2003), allowing for differentiation of capital goods, our model confirms that maintenance and investment appear to be substitutes with respect to the acquisition cost of machines when one assumes a convex cost of maintenance services. Accordingly, the increase in the price of new machines should lead firms to delay replacement and to increase maintenance, thus reducing the rate of depreciation.

To make the further analysis clearer we show that under an additional natural assumption (which, however, is not used in Proposition 7 below) the maintenance of machines of a certain vintage decreases with age.

Lemma 2 Assume that the productivity function $f$ is differentiable, that the depreciation function $\delta(a, m)$ is differentiable in $a$, that $\delta_{m}$ is independent of $a$, and that $\delta_{a}^{\prime}(a, m) \geq 0$ (older machines depreciate faster). Then for the optimal maintenance $m$ and every $t \in[0$, 
$T-\omega]$ and $a \in(0, \omega)$ for which $m(t, a)>0$ it holds that

$$
\frac{\partial m}{\partial a}(t, a) \leq 0
$$

The proof is given in the Appendix. Notice that the particular function $\delta$ defined in (7) apparently satisfies the assumptions of the lemma if $\bar{\delta}$ is differentiable and increasing.

Now we investigate the impact of the rate of the technological progress on the drop of maintenance with age. In order to filter out the role of the other factors, in particular of the productivity level, we consider two identical firms, differing only in the rate of the technological progress of the equipment they use. For the corresponding (differentiable) technology functions, $f_{1}$ and $f_{2}$, we assume that at a time $t^{*} \in[0, T-\omega]$ it holds that $f_{1}\left(t^{*}\right)=f_{2}\left(t^{*}\right)$ (i.e. the firms are competitive), while $f_{1}^{\prime}\left(t^{*}\right)<f_{2}^{\prime}\left(t^{*}\right)$. Denote by $m_{1}$ and $m_{2}$ the corresponding optimal maintenance functions.

Proposition 7 For every $a \in(0, \omega)$ for which $m_{1}\left(t^{*}+a, a\right)>0$ it holds that

$$
\frac{\partial m_{1}}{\partial a}\left(t^{*}+a, a\right)>\frac{\partial m_{2}}{\partial a}\left(t^{*}+a, a\right) .
$$

To avoid misunderstanding, we stress that the functions $m_{1}$ and $m_{2}$ are first differentiated in their second argument, $a$, and then the derivatives are evaluated at $\left(t^{*}+a, a\right)$. The proof of the proposition indicates how the above inequality can be interpreted if some of the derivatives in (18) do not exist.

Proof: Consider ages $a_{1}=a-h$ and $a_{2}=a+h$, where $h>0$ is a small increment. Due to the assumptions that $f_{1}\left(t^{*}\right)=f_{2}\left(t^{*}\right)$ and that $f_{1}^{\prime}\left(t^{*}\right)<f_{2}^{\prime}\left(t^{*}\right)$ we have that $f_{1}\left(t^{*}-h\right)>f_{2}\left(t^{*}-h\right)$ for small $h>0$. Similarly, $f_{1}\left(t^{*}+h\right)<f_{2}\left(t^{*}+h\right)$. Then, according to Proposition 3 , for any $a \in(0, \omega)$ it holds that

$$
\begin{aligned}
m_{1}\left(t^{*}+a, a+h\right)= & m_{1}\left(\left(t^{*}-h\right)+(a+h), a+h\right)>m_{2}\left(\left(t^{*}-h\right)\right. \\
& +(a+h), a+h)=m_{2}\left(t^{*}+a, a+h\right)
\end{aligned}
$$

and similarly $m_{1}\left(t^{*}+a, a-h\right)<m_{2}\left(t^{*}+a, a-h\right)$ (the inequalities are strict where some of the $m$-s are positive). Then

$$
m_{1}\left(t^{*}+a, a+h\right)-m_{1}\left(t^{*}+a, a-h\right)>m_{2}\left(t^{*}+a, a+h\right)-m_{2}\left(t^{*}+a, a-h\right) .
$$

Dividing by $h$ and passing to the limit (where we should notice that the strict inequality is uniform in $h$ ) we complete the proof.

Embodied technological progress is both quantitatively and qualitatively one of the most important features of investment dynamics (see Greenwood et al. 1997). As mentioned in Boucekkine, del Rio, and Martinez (2005), a technological acceleration induces two opposite effects: an incentive to reduce maintenance and scrap earlier to profit from the increased efficiency of new vintages but also as an incentive to increase maintenance and delay scrapping due to a drop in the profitability of investment. The above proposition 
resolves this trade-off analytically so that the maintenance appears to be a substitute of technological growth. Accordingly, the depreciation rate is an increasing function of embodied technological progress and this implies a strong mechanism through which embodiment affects capital depreciation. In particular, one can put forward technological acceleration as the main source of rapid disappearance of early models in the computer industry. It is well known that the surge in computer investment has come as a direct result of rapid price declines, which themselves are a result of rapid technological progress (see, among others, Whelan 2002; Wykoff 2004; Geske, Ramey, and Shapiro 2004). In an attempt to explain how durables with high depreciation rates may be associated with more volatile expenditures, Stacchetti and Stolyarov (2004) state that car bodies are redesigned every 4-5 years and that new generations of Intel processors appear on average every 2-3 years, and computers have higher depreciation rates compared to autos. According to Cummins and Violante (2002), the quality adjusted price fell at an average rate of 23 percent for computers and 2.5 percent for autos each year during 1960-2000. The technological progress in the computer industry has been much more rapid, and it is clear from Proposition 7 that the resources devoted to maintenance would be lower, leading to higher scrapping rates.

So far we have investigated whether investments and maintenance appear as complements or substitutes with respect to variations in different exogenous factors. Now we give an intrinsic definition of complementarity versus substitutability, which is related to the terminology in McGrattan and Schmitz (1999). In contrast to the previous contributions, which are mainly empirical, we present a full analytical characterization aiming to find to what extent maintenance can be a substitute for investment.

Definition 1 Let $I$ and $m$ be the optimal investment and maintenance functions in problem (1)-(6), and let $V(I, m)$ be the corresponding objective value. Maintenance is called a local intrinsic substitute (complement) of investment, if for any smaller investment function $\tilde{I}(t) \leq I(t), t \in[0, T]$ (where the inequality is strict whenever $I(t)>0$ ), it holds that for every maintenance function $\tilde{m}$ for which $\tilde{m}(t, a)>m(t, a)(\tilde{m}(t, a)<m(t, a)$, respectively) whenever $m(t, a)>0$, it holds that

$$
V(\tilde{I}, m+h(\tilde{m}-m))>V(\tilde{I}, m)
$$

for all sufficiently small $h>0$.

Thus $m$ is a local substitute for $I$, if for any downward shifted investment function $\tilde{I}$ (clearly $V(\tilde{I}, m) \leq V(I, m)$ ), it holds that each sufficiently small upward shift of $m$ improves the objective value. Certainly it may happen that maintenance is neither a substitute nor a complement of investment. However, under natural conditions it could be argued that $m$ cannot be both substitute and complement for $I$ (in particular, this follows from the proof of the next proposition).

Proposition 8 Assume that $I(t)>0$ for every $t \in[0, T-\omega)$, and that $m(t, a)$ is not identically zero. Assume also that the marginal cost of maintenance is strictly increasing; that is, $D^{\prime \prime}(M) \geq \rho>0$. Then maintenance is a local intrinsic substitute for investment.

The proof is given in the Appendix. 


\section{Anticipation effects of the technological progress}

As is well known from the work of Jorgenson (1963), a firm's optimal current investment is completely unaffected by the anticipation regarding the future economic environment if the firm can instantaneously and costlessly adjust its capital stock at every point in time. This stems from the fact that the firm's capital stock decision at any instant is independent of decisions made at any other instant. The decision about how much capital to use essentially reduces to a static decision in which the marginal product of capital is equalized to the user cost of capital, implying a myopic investment rule. However, when frictions that prevent instantaneous and costless adjustment of the capital stock are taken into account, the optimality of such a myopic rule ceases to hold (see Gould 1968; Jorgenson 1971; Ando, Modigliani, Rasche, and Turnovsky 1974; and the references therein). In this case, the firm's current choice of technology should then depend on the anticipated forthcoming technological change over the life of the investment as well.

Therefore, our aim in this section is to analyze the question formulated by Chari and Hopenhayn (1991) regarding the anticipation effect: How are decisions to adopt new technologies affected by the prospect that even better technologies will arrive in the future? This question is increasingly important if one has in mind the recent dynamics in the computer industry. Following from figure 5 in Stacchetti and Stolyarov (2004), it is clear that the general pattern seems to be 1 year expenditure spikes (in 1982, 1986, 1991 and 1995) followed by several years of falling investment rates. Apparently, investments are optimally synchronized with the arrival of each new operating system and, accordingly, computer upgrades are delayed until the arrival of the new system. Although many similar examples from different sectors can be found, the analytical characterization of such an anticipation effect of the accelaration in embodied technical progress and the conditions under which it prevails are limited in the published literature.

In a perfectly competitive environment, Feichtinger, Hartl, Kort, and Veliov (2006a) show that future technological developments have no effect on current investment. The firm is assumed to be too small to influence output prices, implying that the revenues of different machines do not influence each other so that current investments do not affect the profitability of investments in future technologies. On the contrary, as shown in Feichtinger, Hartl, Kort, and Veliov (2006b), the situation changes if the firm has market power. A negative anticipation effect occurs so that current investments in recent generations of capital goods decline when faster technological progress is expected to take place in the future. In this case, the firm prefers to delay current investment in new capital goods and invest instead in cheaper old machines with a shorter lifetime that can be scrapped around the time of the technological breakthrough. Similarly, it is proven in Feichtinger, Hartl, Kort, and Veliov (2007) that a negative anticipation effect of technological progress can also prevail as a result of imperfect financial markets. In this case, the firm prefers to acquire financial assets to finance more investments in the more productive machines after the technological breakthrough.

However, these studies that are able to characterize the negative anticipation effect based on the market imperfections assume an exogenous rate of capital depreciation 
and ignore the decision to alter the useful lifetime of the existing capital goods through maintenance services. This ignorance brings with it the following questions: Besides these market imperfections, can an anticipation effect take place as a result of the decisions to alter the useful lifetime of the existing capital goods through maintenance services? Can maintenance services provide another reason to delay current investments in new machines? In reference to these questions, we analytically investigate how an expected technological breakthrough influences the optimal investment and maintenance decisions of a firm.

Following Feichtinger, Hartl, Kort, and Veliov (2006b) we consider the following two technology functions $f$ :

$$
f(s)=f_{1}, \quad \forall s \in[0, T]
$$

and

$$
\bar{f}(s)=\left\{\begin{array}{l}
f_{1} \text { for } s \leq \bar{t}, \\
f_{2} \text { for } s>\bar{t}
\end{array}\right.
$$

where $\bar{t} \in(\omega, T-\omega)$ is a time of a technological breakthrough; ${ }^{3}$ thus $f_{2}>f_{1}$.

We shall compare the optimal behavior of two identical firms, assuming that one of the firms anticipates the technological breakthrough at time $\bar{t}$ from the very beginning of the planning horizon (threfore, it solves its optimization problem with the technology function (19)), whereas the second firm learns about the technology shock when it occurs (therefore, its behavior before time $\bar{t}$ is determined by the technology function (20)).

Denote by $I, m, K, Q, M$ the solution of optimization problem (1)-(6) for the nonanticipating firm (with technology function $f(s)=f_{1}$ ), and by $\bar{I}, \bar{m}, \bar{K}, \bar{Q}, \bar{M}-$ the optimal solution for the anticipating firm (with technology function $\bar{f}$ ).

Our main result in this section is preceded by two lemmas, which are essential for the proof but also are of economic interest.

Lemma 3 claims continuity of the maintenance costs $\bar{M}(t)$ and $M(t)$. The continuity is not an obvious fact, because, as it will be seen in Lemma 4 , the investment, $\bar{I}$, and the maintenance services, $\bar{m}$, of the anticipating firm are discontinuous if $f_{2}>f_{1}$.

Lemma 3 The functions $\bar{M}$ and $\bar{Q}$ are Lipschitz continuous.

\section{Lemma 4}

(i) The optimal investment, $\bar{I}$, is Lipschitz continuous on $[\omega, T-\omega]$, excepting the moment $t=\bar{t}$, where $\bar{I}$ has an upward jump of magnitude at least $c .\left(f_{2}-f_{1}\right)(c$ is a positive constant independent of $f_{1}<f_{2}$, with a bounded $f_{2}$ ).

(ii) The optimal maintenance, $\bar{m}$, is Lipschitz continuous, excepting the segment $\{(\bar{t}+s, s), \quad s \in[0, \omega)\}$, where it has an upward jump of magnitude at least $c .(\omega-s) .\left(f_{2}-f_{1}\right)$ (for those s for which $\left.\bar{m}(\bar{t}+s-0, s)>0\right)$.

\footnotetext{
${ }^{3}$ As before, we exclude from the consideration the initial and the final intervals of length $\omega$ because the behavior of the firm in these intervals is strongly influenced by the initial data and by the end of the planning horizon.
} 
The next proposition claims that the presence of maintenance costs leads to a decrease in investments (negative anticipation effect) before an expected technological breakthrough. To simplify the proof we assume that the maintenance level $m(t, a) \geq 0$ is fixed and the optimization in (1)-(6) is carried out only with respect to the investments $I(t)$.

Proposition 9 Assume that the fixed operation cost, $m_{0}$, is positive, and that the maintenance cost function is marginally increasing: $D^{\prime \prime}(M) \geq \rho>0$. Then $\bar{I}(t)<I(t)$ in some interval contained in $(\bar{t}-2 \omega, \bar{t})$.

The proofs of the above lemmas and proposition are technical and are presented in the Appendix.

Our analysis shows that a negative anticipation effect occurs even for a perfectly competitive firm operating in a perfect financial market if the firm has the freedom to chose the level of maintenance of its machines (hence the scrapping time), under the assumption that the maintenance costs are marginally increasing. As the next experiments show, the negative anticipation effect holds not only for investment, but also for maintenance (if used as a second control variable): the anticipating firm maintains the machines produced before the technological breakthrough less than the non-anticipating firm, and this drop in maintenance begins even before the breakthrough.

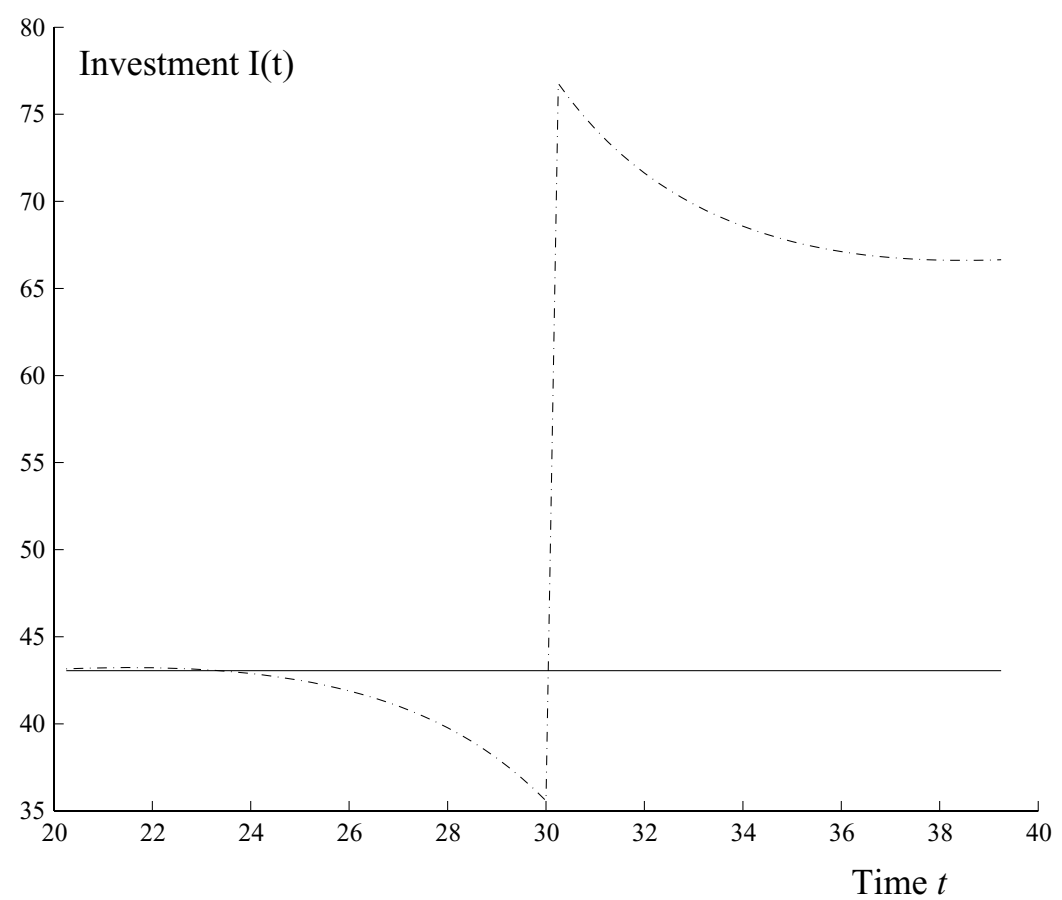

Figure 1 Optimal investment if a technological breakthrough at time $\bar{t}=30$ is anticipated. 


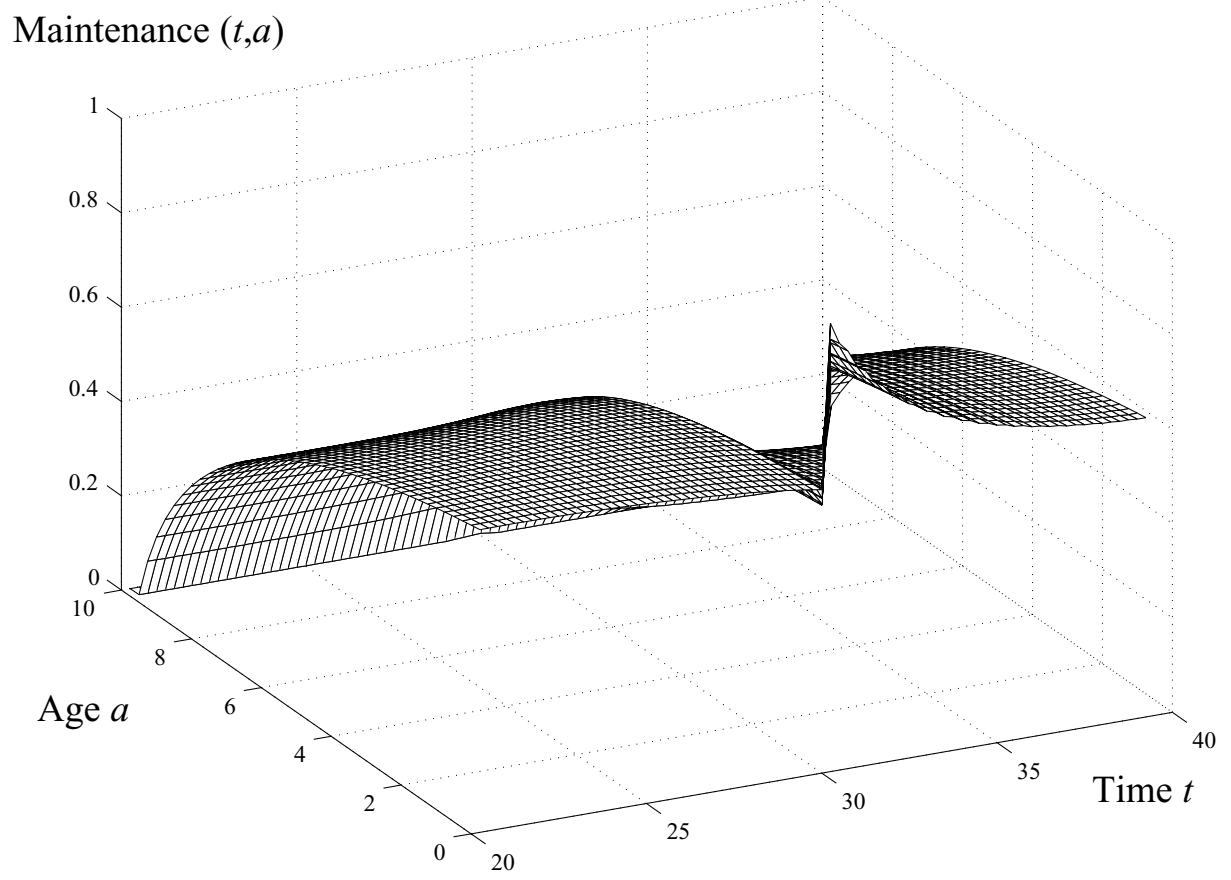

Figure 2 Optimal maintenance if a technological breakthrough at time $\bar{t}=30$ is anticipated.

Figures 1 and 2 represent the optimal investment and maintenance for the anticipating firm with the following data specifications, which are economically plausible: ${ }^{4}$

$$
\begin{aligned}
& p=1 \\
& \omega=10 \text {; } \\
& r=0.003 \text {; } \\
& m_{0}=0.6 \text {; } \\
& C(I)=b I+0.5 c I^{2} \text {, with } b=5, c=1 \text {; } \\
& D(M)=b_{m} M+0.5 c_{m} M^{2} \text {, with } b_{m}=0.6, c_{m}=0.03 \text {; } \\
& \delta(a, m)=\bar{\delta}+1 /(\alpha+\beta m), \bar{\delta}=0.02, \alpha=2, \beta=8 \text {; } \\
& \bar{f}(t) \text { : given by }(20) \text { with } \bar{t}=30, f_{1}=20, f_{2}=30 \text {. }
\end{aligned}
$$

Figure 1 shows the drop of investments before the technological breakthrough at time $\bar{t}=30$ and the jump up at $\bar{t}$. The solid line corresponds to the investment level of the nonanticipating firm before $\bar{t}$. Just before the technological breakthrough the firm reduces the investments in new machines in order to invest more after the breakthrough as it becomes

\footnotetext{
${ }^{4}$ The numerical solution of the of the problem (1)-(6) and of the extended problem considered in the next section are obtained using the general solver developed by the second author, which is presented in Chrissoverghi, Tragler, and Veliov (2007) and Veliov (2003).
} 
more profitable to wait for the new generations endowed with more efficient technologies. The steady level of investment after the shock is higher than that before the shock. The same result applies to the maintenance activities, reinvoking the importance of Propositions 3-5. Figure 2 represents the maintenance services, which also decrease for machines of the old technology even before $\bar{t}$, and jump up for the machines of the new technology (produced after $\bar{t}$ ). It is optimal for the firm to reduce maintenance and let the older vintages scrap earlier just before the shock in order to be able to devote these resources to maintaining the new generations of superior machines after the shock. It is also clear from Figure 2 that maintenance of machines of certain vintage decreases with the age (see Lemma 2). It is remarkable that the machines of superior technology are maintained more shortly after the technological shock than later. In fact, as the investments shortly after $\bar{t}$ overshot the long-run equilibrium corresponding to $f_{2}$, investment and maintenance appear once more as complements.

Remark 6 Clearly, $\bar{f}(t) \geq f(t)$ for every $t$. However, as the above numerical results show (as it is claimed also by Proposition 9 for investments), $\bar{I}(t)<I(t)$ and $\bar{m}(t, a)<m(t, a)$ for some $t$ and $a$. This implies that the assumption of linearity of the maintenance cost is essential for the claim of Proposition 3.

\section{Investments in old technologies}

In the previous sectionswe assumed that the firm can invest only in the newest currently available technology. In this section we allow for investments in old machines, denoted by $J(t, a)$, where $a$ indicates the age of the technology. The possibility to invest in old machines leads to the following changes in the basic model (1)-(6). The equation of the dynamics of the capital becomes

$$
K_{t}+K_{a}=-\delta(a, m(t, a)) K(t, a)+J(t, a), \quad K(0, a)=K_{0}(a), \quad K(t, 0)=I(t) .
$$

The objective function includes the cost of investment in old machines:

$$
\max \int_{0}^{T} e^{-r t}\left[p Q(t)-C(I(t))-D(M(t))-\int_{0}^{\omega} C^{\mathrm{old}}(a, J(t, a)) \mathrm{d} a\right] \mathrm{d} t,
$$

where $C^{\text {old }}(a, J)$ is the cost of installation of $J$ machines of age $a$.

The adjoint equation (8) remains the same as in the case of investments in new machines only. The maximal principle from Feichtinger et al. (2003) implies that

$$
J(t, a)=\left(\left(C^{\text {old }}\right)^{\prime}\right)^{-1}(\xi(t, a)),
$$

where now $J(t, a)$ is allowed to be negative as well. Therefore, a state constraint $K(t, a) \geq 0$ should be added to the model, in principle, but in the numerical results below it is automatically fulfilled.

A remarkable feature of the analysis is that Propositions 3-5 are still valid in the case of investment in old machines. 


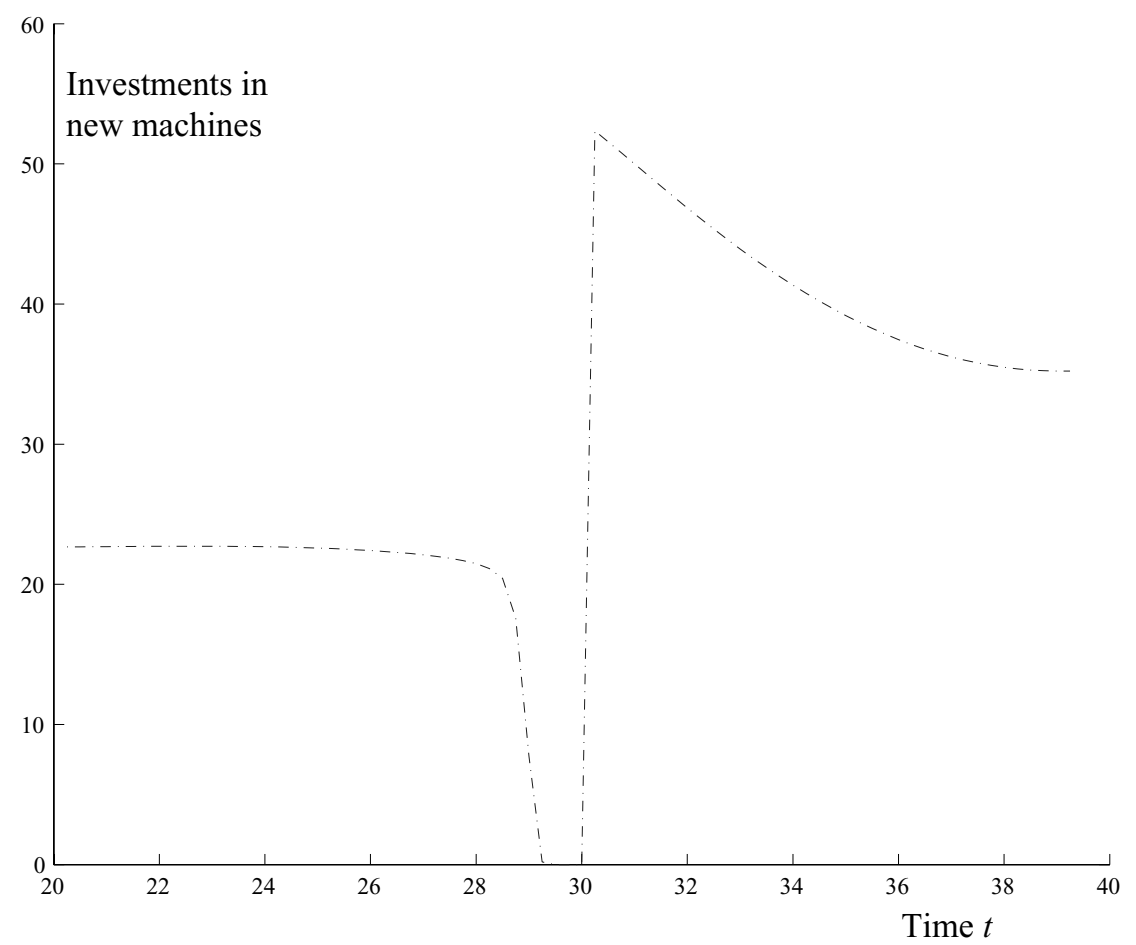

Figure 3 Optimal investment in new machines if a technological breakthrough at time $\bar{t}=30$ is anticipated.

Below we investigate numerically (see footnote 4 ) the impact of the possibility of investing in old machines on the optimal investment and maintenance. We consider a firm with the same parameters as in Section 5 with the additional specification

$$
C^{\text {old }}(a, J)=b^{\text {old }}(a) J+0.5 c^{\text {old }} J^{2}, \quad \text { with } b^{\text {old }}(a)=b *(1-a / \omega), \quad c^{\text {old }}=c .
$$

Figures 3-5 represent the optimal investments in new machines, the optimal investment in old machines, and the optimal maintenance, respectively. Comparing with Figures 1 and 2 we see that the investment in old machines appears as a substitute of both investments in new machines and maintenance. It is remarkable that the relative drop of investment in new machines before the technological shock at $\bar{t}=30$ is larger if investments in old machines are allowed. This is because a part of the investments in new machines shortly before the technological breakthrough at time $\bar{t}$ (see Figure 1) is replaced by investments in old machines, which will be scrapped shortly after $\bar{t}$ so that they can also be replaced by the superior machines. This leads to a second anticipation effect, namely, that there will be more scrapping due to maximal age, $\omega$, soon after $\bar{t}$, compared with the case of $J(t, a)=0$; hence, the still existing machines will be maintained more. This is evident in Figure 5: the peak of $m(t, a)$ for $\bar{t}<t-a<\bar{t}+3$ is much higher than the one on Figure 2, both in relative and in absolute terms. 


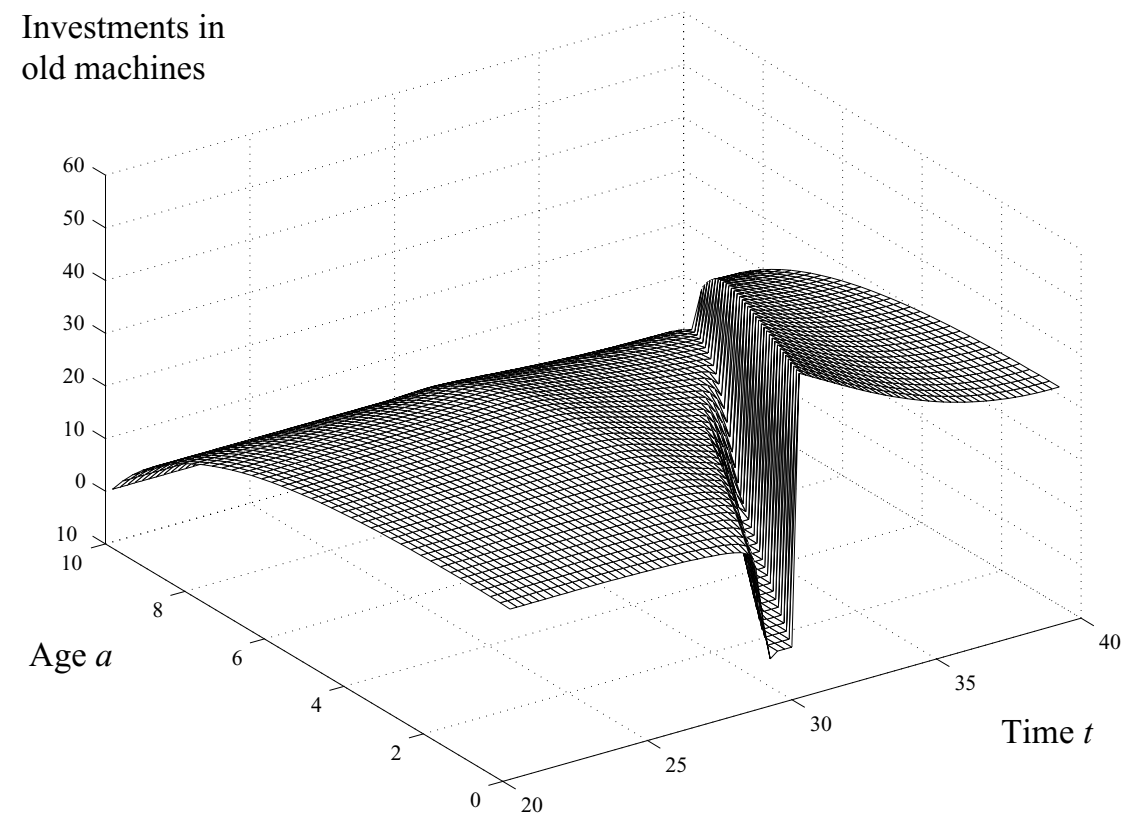

Figure 4 Optimal investment in old machines if a technological breakthrough at time $\bar{t}=30$ is anticipated.

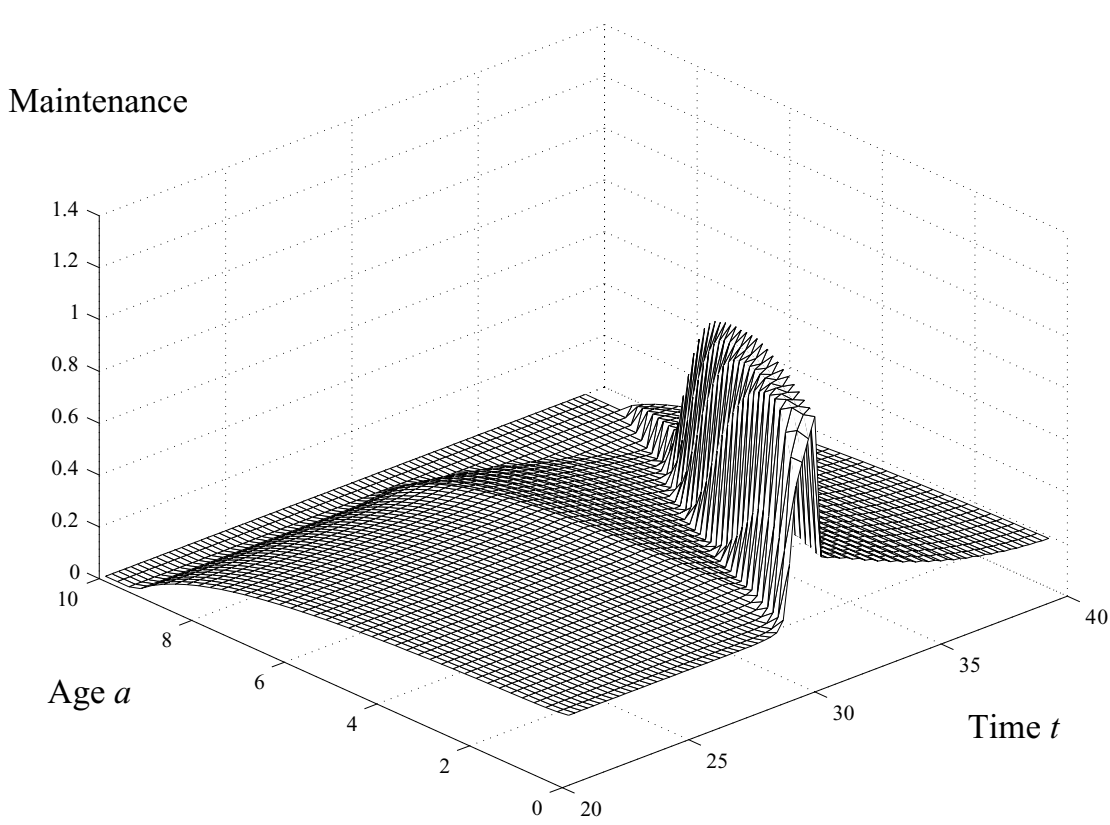

Figure 5 Optimal maintenance if investments in old machines are allowed and if a technological breakthrough at time $\bar{t}=30$ is anticipated. 


\section{Appendix}

Proof of Proposition 1 (A sketch) First of all, it is not difficult to argue that any minimizing sequence $\left\{\left(I_{k}\right.\right.$, $\left.m_{k}\right)$ \} is bounded in $L_{\infty}$ : for $I$, because of the strong convexity of $C$, and for $m$, because of the finite life time of the physical capital and $D^{\prime}(M) \geq \gamma^{D}>0$. Then the main argument is standard, in principle: the $L_{2}$-weak lower semi-continuity of integral functionals with convex integrands.

The function $C(I)$ is supposed convex and $I$ enters linearly in the (boundary condition of) differential equation (2). The function $D(m)$ is also supposed convex, but the main trouble is caused by the nonlinearity of (2) with respect to $m$. However, the solution of (2) has an explicit (Cauchy-type, along the characteristics) representation, where $\delta$ is comfortably integrated. This makes it possible to use the convexity of $\delta$ to prove that the weak limit of $\left\{m_{k}\right\}$ gives an objective value that is not worse than the limit of the objective values provided by $m_{k}$. We skip the details of this proof.

Proof of Lemma 1: On every characteristic line starting at a point $(t, 0)$ (that is on the segment $\{(t+s, s): s \in[0$, $\omega]\})$ the solution $\xi$ of the adjoint equation (8) satisfies an ordinary differential equation. Namely, $\eta(s)=\xi(t+s$, s) satisfies

$$
\eta^{\prime}(s)=(r+\delta(s, m(t+s, s))) \eta(s)+p f(t)-D^{\prime}(M(t+s))\left(m_{0}+m(t+s, s)\right),
$$

with $\eta(\omega)=0$. For $\eta=0$ the right-hand side is

$$
\eta^{\prime}=p f(t)-D^{\prime}(M(t+s))\left(m_{0}+m(t+s, s)\right) .
$$

If it happens that $K(t+s, s)=0$, then $m(t+s, s)=0$ due to the convention made at the end of Section 2 . Otherwise, we also have $m(t+s, s)=0$ due to $(10)$ and the assumption that $D^{\prime}(M(t+s)) \geq \gamma^{D}$. Therefore, we have

$$
\eta^{\prime}=p f(t)-D^{\prime}(M(t+s)) m_{0} \leq p f(t+s)-D^{\prime}(M(t+s)) m_{0}<0
$$

due to the monotonicity of $f$ and the regularity assumption. Therefore, the region $\eta \geq 0$ is invariant with respect to (21) backward in time, and its boundary strictly repels the trajectories.

Proof of Proposition 5: We employ again a comparison argument for the solutions $\eta_{1}$ and $\eta_{2}$ of (16), corresponding to the two functions $\delta_{1}$ and $\delta_{2}$. For any fixed $t \in[0, T-\omega]$ we have to prove that $\eta_{1}(s) \geq \eta_{2}(s)$ for every $s \in[0, \omega]$. Then the claim of the proposition would follow from Remark 4 . That is, we have to prove that if $\eta_{1}=\eta_{2}=\eta>0$ at some point $s$, then $\eta_{1}^{\prime} \leq \eta_{2}^{\prime}$, which is equivalent to

$$
\delta_{1}\left(s, \hat{m}_{1}(s, \eta)\right) \eta+d \hat{m}_{1}(s, \eta) \leq \delta_{2}\left(s, \hat{m}_{2}(s, \eta)\right) \eta+d \hat{m}_{2}(s, \eta)
$$

where

$$
\left.\hat{m}_{i}(s, \eta)\right)=\left(\delta_{i, m}^{\prime}\right)_{+}^{-1}\left(s,-\frac{d}{\eta}\right) .
$$

Substituting $y=-d / \eta$, we have to prove that

$$
\delta_{1}\left(s,\left(\delta_{1, m}^{\prime}\right)_{+}^{-1}(s, y)\right)-\left(\delta_{1, m}^{\prime}\right)_{+}^{-1}(s, y) y \leq \delta_{2}\left(s,\left(\delta_{2, m}^{\prime}\right)_{+}^{-1}(s, y)\right)-\left(\delta_{2, m}^{\prime}\right)_{+}^{-1}(s, y) y
$$

for every $y<0$. To do this we consider three cases.

1. Let $y \leq \delta_{1, m}^{\prime}(s, 0)$. Then, according to condition (ii) $y \leq \delta_{2, m}^{\prime}(s, 0)$. Then 


$$
\left(\delta_{1, m}^{\prime}\right)_{+}^{-1}(s, y)=\left(\delta_{2, m}^{\prime}\right)_{+}^{-1}(s, y)=0,
$$

and (22) is implied by $\delta_{1}(s, 0) \leq \delta_{2}(s, 0)$.

2. Let $\delta_{1, m}^{\prime}(s, 0)<y<\delta_{2, m}^{\prime}(s, 0)$. Then substituting $m_{i}(s, y)=\left(\delta_{i, m}^{\prime}\right)_{+}^{-1}(s, y)$ and taking into account that $\left.y=\delta_{1, m}^{\prime}\left(s, m_{1}(s, y)\right)\right)$ we have

$$
\begin{gathered}
\delta_{1}\left(s,\left(\delta_{1, m}^{\prime}\right)_{+}^{-1}(s, y)\right)-\left(\delta_{1, m}^{\prime}\right)_{+}^{-1}(s, y) y=\delta_{1}\left(s,\left(\delta_{1, m}^{\prime}\right)^{-1}(s, y)\right)-\left(\delta_{1, m}^{\prime}\right)^{-1}(s, y) y \\
\left.=\delta_{1}\left(s, m_{1}(s, y)\right)-\delta_{1, m}^{\prime}\left(s, m_{1}(s, y)\right)\right) m_{1}(s, y) \leq \delta_{1}(s, 0) \\
\quad \leq \delta_{2}(s, 0)=\delta_{2}\left(s,\left(\delta_{2, m}^{\prime}\right)_{+}^{-1}(s, y)\right)-\left(\delta_{2, m}^{\prime}\right)_{+}^{-1}(s, y) y,
\end{gathered}
$$

where the inequality in the second last line follows from the convexity of $\delta_{1}$ with respect to $m$. This also verifies (22) in this case.

3. Let $\delta_{1, m}^{\prime}(s, 0) \leq \delta_{2, m}^{\prime}(s, 0)<y$. In this case, inequality (22) can be equivalently rewritten without the "+" subscripts. Using again the notation $m_{i}(s, y)$ (where now the " + " subscript is omitted), we represent $(22)$ in the following way:

$$
\delta_{1}\left(s, m_{1}(s, y)\right)-y m_{1}(s, y) \leq \delta_{2}\left(s, m_{2}(s, y)\right)-y m_{2}(s, y),
$$

where $\left.\left.y=\delta_{1, m}^{\prime}\left(s, m_{1}(s, y)\right)\right)=\delta_{2, m}^{\prime}\left(s, m_{2}(s, y)\right)\right)$. Then the proposition follows from the following claim.

Claim 1 Let for two differentiable convex functions $g_{1}$ and $g_{2}$, defined on $[0, \infty)$, it hold that $g_{1}(m) \leq g_{2}(m)$ for every $m \geq 0$. Let $m_{1}$ and $m_{2}$ be two points such that $g_{1}^{\prime}\left(m_{1}\right)=g_{2}^{\prime}\left(m_{2}\right)$. Then

$$
g_{1}\left(m_{1}\right)-g_{1}^{\prime}\left(m_{1}\right) m_{1} \leq g_{2}\left(m_{2}\right)-g_{2}^{\prime}\left(m_{2}\right) m_{2} .
$$

The proof is straightforward:

$$
\begin{gathered}
g_{2}\left(m_{2}\right) \geq g_{1}\left(m_{2}\right) \geq g_{1}\left(m_{1}\right)+g_{1}^{\prime}\left(m_{1}\right)\left(m_{2}-m_{1}\right) \\
=g_{1}\left(m_{1}\right)+g_{1}^{\prime}\left(m_{1}\right) m_{2}-g_{1}^{\prime}\left(m_{1}\right) m_{1}=g_{1}\left(m_{1}\right)+g_{2}^{\prime}\left(m_{2}\right) m_{2}-g_{1}^{\prime}\left(m_{1}\right) m_{1},
\end{gathered}
$$

where the first inequality follows from the convexity of $g_{1}$. This implies the claim and, hence, completes the first statement of the proposition.

To prove the second statement of the proposition one should just reconsider the above inequalities bearing in mind the additional assumptions.

Proof of Lemma 2: Because $\delta_{m}$ is independent of $a, \hat{m}$ is also independent of $a$; therefore, below we skip the argument $a$ of $\hat{m}$.

If $m(t, a)>0$ we have

$$
m_{a}(t, a)=\hat{m}_{\xi}(\xi(t, a)) \xi_{a}(t, a) .
$$

Bearing in mind the definition of the function $\hat{m}$ in (13) and applying standard calculus one obtains that

$$
\hat{m}_{\xi}(\xi(t, a))=\left[\delta_{m m}^{\prime \prime}(\hat{m}(\xi))\right]^{-1} \frac{d}{\xi^{2}}>0
$$

where the inequality is due to the standing assumption (ii). Differentiating the adjoint equation (15) in $a$ we obtain for $v(t, a)=\xi_{a}(t, a)$ the equation

$$
v_{t}+v_{a}=[\ldots] v(t, a)+\delta_{a}(a, \hat{m}(\xi(t, a))) \xi(t, a)+p f^{\prime}(t-a), \quad v(t, \omega) \leq 0,
$$


where the term in the brackets is of no importance, and the inequality $v(t, \omega) \leq 0$ follows from $\xi(t, \omega)=0$, $\xi(t, a) \geq 0$. Because $f^{\prime} \geq 0, \delta_{a} \geq 0$, and $\xi(t, a) \geq 0$ (according to Lemma 1), the solution $v(t, a)$ is non-positive, by the same argument used in the proof of Proposition 3 .

Proof of Proposition 8: We consider the control variables $I$ and $m$ as elements of the spaces $L_{\infty}([0, T])$ and $L_{\infty}([0, T] \times[0, \omega])$, respectively. The proof of the maximum principle in Feichtinger et al. (2003) (see also proposition 1 in Veliov (2003)) implies that the functional $m \longrightarrow V(\tilde{I}, m)$ is Gâteaux differentiable and its derivative $d_{m} V$ has a functional representation; namely (compare with the derivative with respect to $m$ of the expression in the right-hand side of (10)),

$$
\mathrm{d}_{m} V(\tilde{I}, m)(t, a)=\left[\tilde{\xi}(t, a) \delta_{m}^{\prime}(a, m(t, a))-D^{\prime}(\tilde{M}(t))\right] \tilde{K}(t, a),
$$

where $\tilde{K}$ and $\tilde{M}$ correspond to the control pair $(\tilde{I}, m)$, and $\tilde{\xi}$ is the corresponding solution of the adjoint equation (8). Clearly, to prove the proposition it is enough to show that $\mathrm{d}_{m} V(\tilde{I}, m)(t, a) \geq 0$, for all $(t, a) \in[0, T-\omega] \times$ $[0, \omega)$ and the inequality is strict whenever $m(t, a)>0$.

Due to the assumption that $I(t)>0$ for all $t<T$, for $\tilde{I}$ as in Definition 1 we have $\tilde{I}(t)>0$; hence, $\tilde{K}(t, a)>0$ for all $t \in[\omega, T)$ and $a \in(0, \omega]$. Then it remains to prove that

$$
\tilde{\xi}(t, a) \delta_{m}^{\prime}(a, m(t, a))-D^{\prime}(\tilde{M}(t)) \geq 0, \quad \text { for }(t, a) \in[0, T-\omega] \times[0, \omega)
$$

and the inequality is strict whenever $m(t, a)>0$.

According to the maximum principle, for $(t, a)$ for which $m(t, a)>0$ we have that

$$
\xi(t, a) \delta_{m}^{\prime}(a, m(t, a))-D^{\prime}(M(t))=0
$$

hence, it suffices to prove the follow two inequalities:

$$
\tilde{\xi}(t, a) \geq \xi(t, a), \quad D^{\prime}(\tilde{M}(t)) \leq D^{\prime}(M(t))
$$

where the latter one is strict for those $t \in[\omega, T)$ for which $m(t, a)>0$ on a set of positive measure. Clearly, for $t$ $\in[\omega, T]$

$$
\tilde{M}(t)=\int_{0}^{\omega}\left(m_{0}+m(t, a)\right) \tilde{K}(t, a) \mathrm{d} a<\int_{0}^{\omega}\left(m_{0}+m(t, a)\right) K(t, a) \mathrm{d} a=M(t),
$$

because obviously $\tilde{K}(t, a)<K(t, a)$ for all $t \in[\omega, T)$ and $a \in(0, \omega]$. Then the condition for $D$ implies $D^{\prime}(M(t)) \geq D^{\prime}(\tilde{M}(t))+\rho(M(t)-\tilde{M}(t))>D^{\prime}(\tilde{M}(t))$. The first inequality in (23) follows from the comparison argument for the solutions of the adjoint equation used several times above. Here the equations for $\tilde{\xi}$ and $\xi$ differ only in the term $D^{\prime}(\tilde{M}(t)) \leq D^{\prime}(M(t))$, which implies the desired inequality.

Proof of Lemma 3: The proof does not use the particular forms of $f$ given by (19) and (20); therefore, we skip the bars in the notations $\bar{M}, \bar{Q}$, etc. The function $\left(\delta_{m}^{\prime}\right)_{+}^{-1}$ is Lipschitz continuous, and the function $\xi \longrightarrow\left(\delta_{m}^{\prime}\right)_{+}^{-1}\left(a,-\frac{D}{\xi}\right)$ is also Lipschitz continuous in $[0,+\infty)$. Moreover, below we use that $\left(\delta_{m}^{\prime}\right)_{+}^{-1}(a, y)$ is monotone increasing in its second argument, and also that the adjoint function $\xi$ is Lipschitz continuous along every characteristic line $\{(t+a, a): a \in[0, \omega]\}$.

Consider $t^{\prime}, t^{\prime \prime} \in[0, T-\omega]$ and let $t^{\prime \prime}=t^{\prime}+\varepsilon$, where $|\varepsilon|$ is a sufficiently small number, so that the manipulations below make sense. Without any restriction we assume that

$$
M\left(t^{\prime}\right) \leq M\left(t^{\prime \prime}\right)
$$


Furthermore, $c_{1}, c_{2}, \ldots$ denote constants that are independent of $t^{\prime}$ and $t^{\prime \prime}$. We have

$$
\begin{aligned}
m\left(t^{\prime}, a\right) & =\left(\delta_{m}^{\prime}\right)_{+}^{-1}\left(a,-\frac{D^{\prime}\left(M\left(t^{\prime}\right)\right)}{\xi\left(t^{\prime}, a\right)}\right) \geq\left(\delta_{m}^{\prime}\right)_{+}^{-1}\left(a,-\frac{D^{\prime}\left(M\left(t^{\prime \prime}\right)\right)}{\xi\left(t^{\prime}, a\right)}\right) \\
& \geq\left(\delta_{m}^{\prime}\right)_{+}^{-1}\left(a,-\frac{D^{\prime}\left(M\left(t^{\prime \prime}\right)\right)}{\xi\left(t^{\prime \prime}, a+\varepsilon\right)}\right)-c_{1}|\varepsilon| \geq\left(\delta_{m}^{\prime}\right)_{+}^{-1}\left(a+\varepsilon,-\frac{D^{\prime}\left(M\left(t^{\prime \prime}\right)\right)}{\xi\left(t^{\prime \prime}, a+\varepsilon\right)}\right)-c_{2}|\varepsilon| \\
& =m\left(t^{\prime \prime}, a+\varepsilon\right)-c_{2}|\varepsilon| .
\end{aligned}
$$

Using this inequality and the Lipschitz continuity of $K$ along every characteristic line $\{(t+a, a): a \in[0, \omega]\}$, we obtain that

$$
\begin{aligned}
M\left(t^{\prime \prime}\right) & =\int_{0}^{\omega} m_{0} K\left(t^{\prime \prime}, a\right) \mathrm{d} a+\int_{0}^{\omega} m\left(t^{\prime \prime}, a\right) K\left(t^{\prime \prime}, a\right) \mathrm{d} a \\
& \leq \int_{\varepsilon}^{\omega-\varepsilon} m_{0} K\left(t^{\prime \prime}, a\right) \mathrm{d} a+\int_{\varepsilon}^{\omega-\varepsilon} m\left(t^{\prime \prime}, a\right) K\left(t^{\prime \prime}, a\right) \mathrm{d} a+c_{3}|\varepsilon| \\
& =\int_{\varepsilon}^{\omega-\varepsilon} m_{0} K\left(t^{\prime}+\varepsilon, a\right) \mathrm{d} a+\int_{0}^{\omega-2 \varepsilon} m\left(t^{\prime \prime}, a+\varepsilon\right) K\left(t^{\prime \prime}, a+\varepsilon\right) \mathrm{d} a+c_{3}|\varepsilon| \\
& \leq \int_{\varepsilon}^{\omega-\varepsilon} m_{0} K\left(t^{\prime}, a-\varepsilon\right) \mathrm{d} a+\int_{0}^{\omega-2 \varepsilon}\left(m\left(t^{\prime}, a\right)+c_{2}|\varepsilon|\right) K\left(t^{\prime}, a\right) \mathrm{d} a+c_{4}|\varepsilon| \\
& \leq \int_{0}^{\omega-2 \varepsilon} m_{0} K\left(t^{\prime}, a\right) \mathrm{d} a+\int_{0}^{\omega-2 \varepsilon} m\left(t^{\prime}, a\right) K\left(t^{\prime}, a\right) \mathrm{d} a+c_{5}|\varepsilon| \\
& \leq M\left(t^{\prime}\right)+c_{6}|\varepsilon| .
\end{aligned}
$$

Taking into account (24) we obtain that

$$
\left|M\left(t^{\prime \prime}\right)-M\left(t^{\prime}\right)\right| \leq c_{6}\left|t^{\prime}-t^{\prime \prime}\right|
$$

which proves the first claim of the lemma.

To prove the Lipschitz continuity of $Q$ it is enough to use again the Lipschitz continuity of $K$ along the characteristic lines. Indeed, with the standard use of $O(h)$ we have for any sufficiently small $h$ that

$$
\begin{gathered}
Q(t+h)=\int_{0}^{\omega} f(t+h-a) K(t+h, a) \mathrm{d} a \\
\left.=\int_{0}^{\omega} f(t-(a-h))\right) K(t, a-h) \mathrm{d} a+O(h) \\
\left.\int_{-h}^{\omega-h} f(t-a)\right) K(t, a) \mathrm{d} a+O(h)=\int_{0}^{\omega} f(t-a) K(t, a) \mathrm{d} a+O(h)=Q(t)+O(h) .
\end{gathered}
$$

This completes the proof of the lemma.

It is useful to note that the Lipschitz constants are uniform in $f$ when $f$ is bounded.

Proof of Lemma 4: We consider again the function $\eta(s)=\xi(t+s, s)$ (where $t \leq T-\omega$ is fixed), which satisfies (21) with $\eta(\omega)=0$. From the maximum principle we have that

$$
m(t+s, s)=\left(\delta_{m}^{\prime}\right)_{+}^{-1}\left(s,-\frac{D^{\prime}(M(t+s))}{\eta(s)}\right):=\mu(t+s, s, \eta(s)) .
$$

Thus $\eta$ also satisfies the equation

$$
\begin{aligned}
\eta^{\prime}(s)= & (r+\delta(s, \mu(t+s, s, \eta(s)))) \eta(s) \\
& -p f(t)+D^{\prime}(M(t+s))\left(m_{0}+\mu(t+s, s, \eta(s))\right) .
\end{aligned}
$$


Now we fix two different values $t=t_{1}<\bar{t}$ and $t=t_{2}>\bar{t}$ and denote by $\eta_{1}$ and $\eta_{2}$ the corresponding solutions of the above equation. Notice that we have $f\left(t_{1}\right)=f_{1}$ and $f\left(t_{2}\right)=f_{2}$.

Now we shall estimate the difference $\dot{\eta}_{2}(s)-\dot{\eta}_{1}(s)$ for those $s$ for which $\eta_{2}(s) \geq \eta_{1}(s)$. In the estimation we use that $\mu$ is Lipschitz and monotone increasing with respect to $\eta$ (see the end of Section 3), that $M$ is Lipschitz (see Lemma 3), and that, as a consequence of the latter, $\mu$ is Lipschitz with respect to $t$. Then

$$
\begin{aligned}
& \dot{\eta}_{2}(s)-\dot{\eta}_{1}(s) \\
& \quad=\left[\left(r+\delta\left(s, \mu\left(t_{2}+s, s, \eta_{2}(s)\right)\right) \eta_{2}(s)\right.\right. \\
& \quad-\left(r+\delta\left(s, \mu\left(t_{1}+s, s, \eta_{2}(s)\right)\right) \eta_{2}(s)\right]-p\left(f_{2}-f_{1}\right) \\
& \quad+\left[D^{\prime}\left(M\left(t_{2}+s\right)\right)\left(m_{0}+\mu\left(t_{2}+s, s, \eta_{2}(s)\right)\right)\right. \\
& \left.\quad-D^{\prime}\left(M\left(t_{1}+s\right)\right)\left(m_{0}+\mu\left(t_{1}+s, s, \eta_{1}(s)\right)\right)\right] \\
& \quad \leq\left[r+\delta\left(s, \mu\left(t_{1}+s, s, \eta_{1}(s)\right)\right)\right]\left(\eta_{2}-\eta_{1}\right) \\
& \quad+\left[\delta\left(s, \mu\left(t_{1}+s, s, \eta_{2}(s)\right)\right)-\delta\left(s, \mu\left(t_{1}+s, s, \eta_{1}(s)\right)\right)\right] \eta_{2}(s) \\
& \quad+c_{1}\left(t_{2}-t_{1}\right)-p\left(f_{2}-f_{1}\right) \\
& \left.\left.\quad+D^{\prime}\left(M\left(t_{1}+s\right)\right)\left[\mu\left(t_{1}+s, s, \eta_{2}(s)\right)\right)-\mu\left(t_{1}+s, s, \eta_{1}(s)\right)\right)\right]+c_{2}\left(t_{2}-t_{1}\right),
\end{aligned}
$$

where $c_{1}, c_{2}, \ldots$ are independent of $t_{1}, t_{2}, f_{1}$ and $f_{2}$ (for $f_{1} \leq f_{2}$ taking values in a compact interval). Given that $\mu$ is increasing in $\eta$ and $\delta$ is decreasing in $m$ we observe that the expression in the second brackets is non-positive, whereas the one in the last brackets is non-negative. Then due to the monotonicity and Lipschitz continuity of $\mu$

$$
\begin{aligned}
\dot{\eta}_{2}(s) & -\dot{\eta}_{1}(s) \leq\left[r+\delta\left(s, \mu\left(t_{1}+s, s, \eta_{1}(s)\right)\right)\right]\left(\eta_{2}-\eta_{1}\right) \\
& -p\left(f_{2}-f_{1}\right)+c_{4}\left(\eta_{2}-\eta_{1}\right)+c_{3}\left(t_{2}-t_{1}\right) .
\end{aligned}
$$

Because $\delta$ is positive and bounded we obtain that for $s$ for which $\eta_{2}(s) \geq \eta_{1}(s)$ it holds that

$$
\dot{\eta}_{2}(s)-\dot{\eta}_{1}(s) \leq c_{5}\left(\eta_{2}(s)-\eta_{1}(s)\right)-p\left(f_{2}-f_{1}\right)+c_{3}\left(t_{2}-t_{1}\right)
$$

Because $\eta_{1}(\omega)=\eta_{2}(\omega)=0$, and because $p\left(f_{2}-f_{1}\right)>c_{3}\left(t_{2}-t_{1}\right)$ for $t_{1}$ and $t_{2}$ sufficiently close to $\bar{t}$ we conclude that $\eta_{2}(s)>\eta_{1}(s)$ for every $s \in[0, \omega)$. Moreover, from the Cauchy formula,

$$
\begin{gathered}
\eta_{2}(s)-\eta_{1}(s) \geq\left[p\left(f_{2}-f_{1}\right)-c_{3}\left(t_{2}-t_{1}\right)\right] \int_{s}^{\omega} e^{-c_{5}(\theta-s)} \mathrm{d} s \\
\geq c_{6}\left[p\left(f_{2}-f_{1}\right)-c_{3}\left(t_{2}-t_{1}\right)\right](\omega-s) .
\end{gathered}
$$

Then the claims of the lemma follow from the representations of the optimal controls by the formulas (12).

The claims about Lipschitz continuity follow from the same argument, because the term $p\left(f_{2}-f_{1}\right)$ disappears if $t_{1}$ and $t_{2}$ are from the same side of $\bar{t}$.

Proof of Proposition 9: Because we know that $\bar{I}$ and $I$ are continuous in $(\bar{t}-2 \omega, \bar{t})$, it is enough to prove that $\bar{I}(t)<I(t)$ for some $t$. Assume that $\bar{I}(t) \geq I(t)$ for all $t$ from this interval. Then (due to the assumption that $\bar{m}=m) \bar{K}(t+a, a) \geq K(t+a, a)$ for all $t \in(\bar{t}-\omega, \bar{t}]$ and $a \in[0, \omega]$. This implies

$$
\bar{M}(t) \geq M(t) \text { for } t \in[\bar{t}-\omega, \bar{t}],
$$

and also

$$
\bar{K}(t, a) \geq K(t, a) \quad \text { for } t \in[\bar{t}, \bar{t}+\omega], \quad a \in[t-\bar{t}, \omega] .
$$

On the other hand, according to Lemmas 3 and 4 we have for $t>\bar{t}$

$$
\begin{aligned}
\bar{I}(t) \geq & \bar{I}(\bar{t}+0)-c_{1}(t-\bar{t}) \geq \bar{I}(\bar{t}-0)+c_{2}\left(f_{2}-f_{1}\right)-c_{1}(t-\bar{t}) \\
& \geq I(\bar{t}-0)+c_{2}\left(f_{2}-f_{1}\right)-c_{1}(t-\bar{t}) \geq I(t)+c_{2}\left(f_{2}-f_{1}\right)-c_{3}(t-\bar{t}),
\end{aligned}
$$


where $c_{1}, c_{2}, \ldots$ are appropriate constants. Thus for a $t^{*}>\bar{t}$ that is sufficiently close to $\bar{t}$ and for some $\gamma>0$ it holds that

$$
\bar{I}(t) \geq I(t)+\gamma \text { for } t \in\left(\bar{t}, t^{*}\right) .
$$

As before, this implies

$$
\bar{K}(t, a) \geq K(t, a)+c_{4} \gamma \quad \text { for } t \in\left[\bar{t}, t^{*}\right], \quad a \in[0, t-\bar{t}] .
$$

Using this inequality and (25) we obtain that for $t \in\left[\bar{t}, t^{*}\right]$

$$
\begin{aligned}
\bar{M}(t) & =\int_{0}^{t-\bar{t}}\left[m_{0}+m(t, a)\right] \bar{K}(t, a) \mathrm{d} a+\int_{t-\bar{t}}^{\omega}\left[m_{0}+m(t, a)\right] \bar{K}(t, a) \mathrm{d} a \\
& \geq \int_{0}^{t-\bar{t}}\left[m_{0}+m(t, a)\right]\left[K(t, a)+c_{4} \gamma\right] \mathrm{d} a+\int_{t-\bar{t}}^{\omega}\left[m_{0}+m(t, a)\right] K(t, a) \mathrm{d} a \\
& =M(t)+\int_{0}^{t-\bar{t}}\left[m_{0}+m(t, a)\right] c_{4} \gamma \mathrm{d} a \geq M(t)+c_{5}(t-\bar{t}) .
\end{aligned}
$$

To complete the proof it is enough to consider (21) for the anticipating and for the non-anticipating firm for $t \in\left(\bar{t}-\omega, t^{*}-\omega\right)$. Because we obtained that $\bar{M}(t+s) \geq M(t+s)$ and the inequality is strict for $s>\bar{t}-t$, and because $f(t)=f_{1}$ in both cases, we conclude that the solution $\bar{\eta}$ for the anticipating firm, and $\eta$ for the non-anticipating one, satisfy

$$
\bar{\eta}(0)<\eta(0),
$$

which implies $\bar{I}(t)<I(t)$ according to (12). The proof is complete.

\section{References}

Ando, A. K., F. Modigliani, R. Rasche, and S. J. Turnovsky (1974), "On the role of expectations of price and technological change in an investment," International Economic Review 15, 384-414.

Aseev, S. M., and A. V. Kryazhimskiy (2004), "The Pontryagin maximum principle and transversality conditions for a class of optimal control problems with infinite time horizons," SIAM Journal of Control Optimization 43, 1094-119.

Aubin, J.-P., and F. Clarke (1979), "Shadow prices and duality for a class of optimal control problems," SIAM Journal Control Optimization 19, 567-86.

Barucci, E., and F. Gozzi (1998), "Investment in a vintage capital model," Research in Economics 52, 159-88.

Benhabib, J., and A. Rustichini (1991), "Vintage capital, investment and growth," Journal of Economic Theory 55, 323-39.

Berkovec, J. (1985), "New car sales and used car stocks: A model of the automobile market," RAND Journal of Economics 16, 195-214.

Bitros, G. C. (1976), "A statistical theory of expenditures in capital maintenance and repair," The Journal of Political Economy 84, 917-36.

Bitros, G. C., and H. Kelejian (1974), "On the variability of the replacement investment capital stock ratio: Some evidence from capital scrappage," The Review of Economics and Statistics 56, 270-78.

Boucekkine, R., D. De la Croix, and O. Licandro (2004), "Modelling vintage structures with DDEs: Principles and applications," Mathematical Population Studies 11, 151-79. 
Boucekkine, R., D. De la Croix, and O. Licandro (2008), "Vintage capital," S. N. Durlauf, and Lawrence E. Blume, eds, New Palgrave Dictionary in Economics, London: Palgrave Macmillan.

Boucekkine, R., F. del Rio, and B. Martinez (2005), “Technological progress and depreciation,” Working Paper No. WP-AD 2005-22, Instituto Valenciano de Investigaciones Economicas.

Boucekkine, R., B. Martinez, and C. Saglam (2006), "Capital maintenance vs technology adoption under embodied technical progress," Contributions in Macroeconomics, The B.E. Journal of Macroeconomics 6, Article 7.

Boucekkine, R., M. Germain, and O. Licandro (1997), "Replacement echoes in the vintage capital growth models," Journal of Economic Dynamics and Control 19, 711-34.

Boucekkine, R., M. Germain, O. Licandro, and A. Magnus (1998), "Creative destruction, investment volatility and the average age of capital," Journal of Economic Growth 3, 361-84.

Boucekkine, R., and R. Ruiz-Tamarit (2003), “Capital maintenance and investment: Complements or Substitutes?” Journal of Economics 78, 1-28.

Brokate, M. (1985), “Pontryagin's principle for control problems in age-dependent population dynamics” Journal of Mathematical Biology 23, 75-101.

Chari, V. V., and H. Hopenhayn (1991), "Vintage human capital, growth, and the diffusion of new technology," Journal of Political Economy 99, 1142-65.

Chrissoverghi, J., G. Tragler, and V. M. Veliov (2007), "Numerical simulation and optimal control of age- and size-structured systems," forthcoming.

Collard, F., and T. Kollintzas (2000), "Maintenance, utilization and depreciation along the business cycle," Discussion Paper 2477, Center for Economic Policy Research, London, UK.

Cummins, J. G., and G. L. Violante (2002), "Investment specific technical change in the United States (1947-2000): Measurement and macroeconomic consequences," Review of Economic Dynamics 5, 243-84.

Dekle, R. (2001), “A note on growth with vintage capital,” Economics Letters 72, 263-7.

Feichtinger, G., R. F. Hartl, P. M. Kort, and V. M. Veliov (2006a), "Capital accumulation under technological progress and learning: A vintage capital approach,” European Journal of Operations Research 172, $293-310$.

Feichtinger, G., R. F. Hartl, P. M. Kort, and V. M. Veliov (2006b), “Anticipation effects of technological progress on capital accumulation: A vintage capital approach," Journal of Economic Theory 126, 143-64.

Feichtinger, G., R. F. Hartl, P. M. Kort, and V. M. Veliov (2007), "Financially constrained capital investments: The effects of disembodied and embodied technological progress," Journal of Mathematical Economics 44, 459-83.

Feichtinger, G., G. Tragler, and V. M. Veliov (2003), "Optimality conditions for age-structured control systems," Journal of Mathematical Analysis and Applications 288, 47-68.

Feichtinger, G., and V. M. Veliov (2007), "On a distributed control problem arising in dynamic optimization of a fixed-size population," SIAM Journal of Optimization 18, 980-1003.

Geske, M., V. Ramey, and M. Shapiro (2004), “Why do computers depreciate?” Working Paper 10831, National Bureau of Economic Research, Cambridge, MA.

Gordon, R. J. (1990), The Measurement of Durable Goods Prices, Chicago: University of Chicago Press.

Gould, J. P. (1968), “Adjustment costs in the theory of investment of the firm," Review of Economic Studies 35, 47-55.

Greenwood, J., Z. Hercowitz, and P. Krussel (1997), “Long-run implications of investment-specific technological change," The American Economic Review 87, 342-62.

Grilliches, Z. (1960), “Measuring inputs in agriculture: A critical survey,” Journal of Farm Economics, 42, 1411427.

Gylfason, T., and G. Zoega (2001), “Obsolescence,” Discussion Paper 2833, Center for Economic Policy Research, London, UK.

Hamilton, B. W., and M. K. Macauley (1999), "Heredity or environment: Why is automobile longevity increasing?" The Journal of Industrial Economics 47, 251-61.

Hartl, R. (1983), “Optimal maintenance and production rates for a machine," Journal of Economic Dynamics and Control 6, 281-306.

Hulten, C. R., and F. C. Wykoff (1995), “Issues in the measurement of economic depreciation," Economic Inquiry $34,10-23$. 
Johansen, L. (1959), "Substitution versus fixed proportion coefficients in the theory of capital growth," Econometrica 27, 1157-76.

Jorgenson, D. W. (1963), “Capital theory and investment behavior,” The American Economic Review 53, $247-59$.

Jorgenson, D. W. (1971), “Econometric studies of investment behavior: A survey,” Journal of Economic Literature 9, 1111-47.

Laitner, J., and D. Stolyarov (2003), “Technological change and the stock market,” The American Economic Review 93, 1240-67.

Licandro, O., and L. Puch (2000), "Capital utilization, maintenance costs and the business cycle," Annales d'Economie et Statistique 58, 143-64.

Malcomson, J. (1975), "Replacement and the rental value of capital equipment subject to obsolescence," Journal of Economic Theory 10, 24-41.

McGrattan, E., and J. Schmitz (1999), "Maintenance and repair: Too big to ignore," Federal reserve Bank of Minneapolis Quarterly Review 23, 2-13.

Mukoyama, T. (2007), "Endogenous depreciation, mismeasurement of aggregate capital, and the productivity slowdown," Journal of Macroeconomics, forthcoming.

Mullen, J. K., and M. Williams (2004), "Maintenance and repair expenditures: Determinants and tradeoffs with new capital goods," Journal of Economics and Business 56, 483-99.

Nelson, R., and M. Caputo (1997), "Price changes, maintenance, and the rate of depreciation," Review of Economics and Statistics 79, 422-30.

Parks, R. (1977), “Determinants of scrappage rates for postwar vintage automobiles,” Econometrica 45, $1099-116$.

Parks, R. (1979), “Durability, maintenance and the price of used assets,” Economic Inquiry 17, 197-217.

Schmalensee, R. (1974), "Market structure, durability, and maintenance effort," Review of Economic Studies 41, 277-87.

Solow, R. (1960), “Mathematical methods in the social sciences, 1959,” K. J. Arrow, S. Karlin, and P. Suppes, eds, Investment and Technological Progress, 89-104. Stanford: Stanford University Press.

Solow, R., J. Tobin, C. C. Von Weizsacker, and M. Yaari (1966), "Neoclassical growth with fixed factor proportions," Review of Economic Studies 33, 79-115.

Stacchetti, E., and D. Stolyarov (2004), "Obsolescence of durable goods and optimal consumption," Mimeo, University of Michigan, Ann Arbor, MI.

Veliov, V. M. (2003), “Newton's method for problems of optimal control of heterogeneous systems,” Optimization Methods and Software 18, 689-703.

Webb, G. F. (1985), Theory of Nonlinear Age-dependent Population Dynamics, New York: Marcel Dekker.

Whelan, K. (2002), "Computers, obsolescence and productivity," The Review of Economics and Statistics 84, 445-61.

Wykoff, F. C. (2004), “Obsolescence vs. deterioration with embodied technological change,” Mimeo, Pomona College, Claremont, CA. 